\title{
Exploring extra dimensions through inflationary tensor modes
}

\section{Sang Hui Im, Hans Peter Nilles and Andreas Trautner}

Bethe Center for Theoretical Physics and Physikalisches Institut der Universität Bonn, Nussallee 12, 53115 Bonn, Germany

E-mail: shim@th.physik.uni-bonn.de, nilles@th.physik.uni-bonn.de, atrautner@uni-bonn.de

AbStract: Predictions of inflationary schemes can be influenced by the presence of extra dimensions. This could be of particular relevance for the spectrum of gravitational waves in models where the extra dimensions provide a brane-world solution to the hierarchy problem. Apart from models of large as well as exponentially warped extra dimensions, we analyze the size of tensor modes in the Linear Dilaton scheme recently revived in the discussion of the "clockwork mechanism". The results are model dependent, significantly enhanced tensor modes on one side and a suppression on the other. In some cases we are led to a scheme of "remote inflation", where the expansion is driven by energies at a hidden brane. In all cases where tensor modes are enhanced, the requirement of perturbativity of gravity leads to a stringent upper limit on the allowed Hubble rate during inflation.

Keywords: Brane Dynamics in Gauge Theories, Cosmology of Theories beyond the SM, Large Extra Dimensions, Spacetime Singularities

ARXiv EPrint: 1707.03830 


\section{Contents}

1 Introduction 1

2 General considerations 3

2.1 Metric and expansion law 3

2.2 Effective Planck mass during inflation 5

2.3 Tensor modes in braneworld inflation 6

3 Large extra dimensions and Randall-Sundrum scenario $\quad 6$

$\begin{array}{ll}3.1 \text { General form of the metric warping } & 6\end{array}$

$\begin{array}{ll}3.2 & \text { Solution for the metric during inflation }\end{array}$

$\begin{array}{lll}3.2 .1 & \text { Large extra dimensions } & 7\end{array}$

$\begin{array}{lll}3.2 .2 & \text { Randall-Sundrum scenarios } & 9\end{array}$

4 Linear dilaton model $\quad 18$

$\begin{array}{llr}4.1 & \text { Generalities } & 18\end{array}$

$\begin{array}{lll}4.2 & \text { The static case } & 19\end{array}$

4.3 The expanding case with external stabilization 20

4.4 The expanding case with dilaton stabilization 23

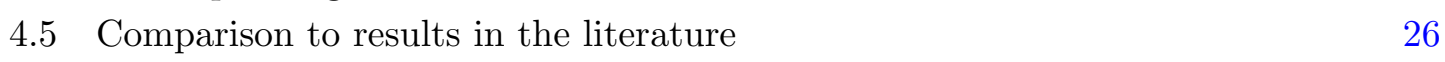

5 Conclusions $\quad 26$

$\begin{array}{lll}\text { A Agreement with earlier results on the RS1 case } & 27\end{array}$

B Dictionary of different coordinate conventions 28

\section{Introduction}

Combining the presence of extra space dimensions with an inflationary phase of the early universe might lead to novel and testable insights in cosmology. Extra dimensions arise in unified schemes like superstring theory and could provide solutions of the weak scale hierarchy problem. Predictions of the inflationary scheme such as e.g. the fluctuations in the cosmic microwave background test the situation at highest cosmological energies and might be influenced by the presence of extra dimensions. Setting up an inflationary scheme in higher dimensions is a challenge as it would have to provide a solution to the moduli stabilization problem and also explain why some space dimensions expand while others are fixed in size. The question of the nature of the inflaton field (is it a brane or a bulk field) would also have to be addressed. 
Up to now, work in this direction concentrated on a very specific picture, which we call the IRB assumption. It assumes that inflation is driven by a field on our visible brane and assumes that radii of extra dimensions are fixed by a separate mechanism that does not influence the specific prediction of the inflationary scheme. Even with this simplified assumption there is an impact of the presence of the extra dimensions: gravity could have a different strength in the bulk and influence the size of tensor modes in the inflationary model under consideration [1] (cf. also [2,3], and [4, ch. 5.1] for a review). This is particularly interesting in models that try to solve the weak scale hierarchy by large or warped extra dimensions. In this case, matter and inflaton field live on our visible infrared (IR) brane while gravity is stronger in the bulk and at a hidden ultraviolet (UV) brane. ${ }^{1}$ Work along these directions has been done in the framework of large extra dimensions (LED) $[5,6]$ and warped extra dimensions à la Randall-Sundrum (RS) [7].

The present work has its origin in the study of inflationary models within the so-called Linear Dilaton model (LD) [8-11] which regained popularity from a discussion of aligned axions $[12,13]$ and the clockwork scheme [14-18]. It can accommodate a solution of the weak-scale hierarchy problem in a braneworld scenario (with IR- and UV-brane) with power law warping (in contrast to exponential warping in the RS case). When studying the LD model within the framework of the simplifying IRB assumption we were led to some inconsistencies to be explained later. To achieve the standard inflationary picture on the visible brane some contributions from the invisible brane (or bulk) are needed. This observation leads us to reconsider a more general picture of extra-dimensional inflation beyond the simplest assumption (both in the LD and the RS model) and this is a main subject of this paper.

Our discussion is organized as follows. In section 2 we will summarize the formulae relevant for the discussion. As a warm-up, we then repeat the discussion for the LED case with one extra dimension using the simplifying IRB assumption. In this case we find an enhancement of tensor modes as the effective Planck mass is reduced through extra dimensional effects. There is an upper bound on the Hubble scale during inflation as well as on the reheating temperature after inflation. Still in some regions of parameter space one could find a sizable tensor-to-scalar ratio due to the transdimensional enhancement. We then turn to the RS model and consider inflation under the simplifying IRB assumption. Again the Planck mass is reduced during inflation, implying that the strength of gravity is enhanced. The tensor-to-scalar ratio is enhanced and we obtain an upper bound on the Hubble parameter. This reproduces known results in the literature based on the simplified assumptions [1].

Next we turn our attention to a wider class of inflationary solutions. We assume a two-brane RS model with our matter on the IR brane. The exponential warping of the extra dimension could explain the weakness of gravity on the visible brane and thus solve the hierarchy problem. Even in the static case we see that the properties of the system strongly depend on the physics at both branes: IR and UV. The radius of the extra

\footnotetext{
${ }^{1}$ Concerning the terminology in this work, we will always refer to our brane - at which the Standard Model lives - as the visible brane and place it at the origin $(z=0)$ of any extra-dimensional coordinate. Irrespectively, we refer to the IR brane as one at which the weak scale hierarchy problem is solved, and to the UV brane as one at which the hierarchy problem is not solved. In this sense, the visible brane coincides with the IR brane if our weak scale hierarchy problem is solved by the presence of an extra dimension.
} 
dimension can be tuned through a choice of the brane tensions on the visible and hidden brane. The implications of energy on the two branes are highly interdependent. A model where inflation is driven originally by inflationary dynamics at the visible brane could be made static by tuning the energy density of the hidden brane. Alternatively the physics at the hidden brane could be the only source of inflationary behavior, a phenomenon one might call "remote" inflation. In this case the Planck mass is enhanced during inflation implying that the strength of gravity is reduced compared to the static case. We also treat a specific case in this general class of solutions discussed earlier by Nihei-Kaloper-Kim-Kim (NKKK) [19-21] as well as the single brane warped model RS2 [22].

In section 4 we consider the Linear Dilaton model. As in RS we have a negative cosmological constant in the bulk and two branes (with matter fields and inflaton field on the visible IR brane). A hierarchy of scales appears because of a power-law warping (in contrast to exponential warping in the RS-case). This situation is more complicated as there is an additional degree of freedom in the bulk (the dilaton). Despite of this we can derive the solution for the static case $(H=0)$ in full generality.

Unfortunately this is no longer true in the expanding case $H \neq 0$. There we perform a perturbative expansion in $H^{2}$. We again adopt the simplifying IRB assumption that we can ignore specific properties of the stabilization process (imposed by an external mechanism). The study of this naive case leads to the amazing result that we are not able to recover the standard inflationary expansion on the visible brane (in contrast to the RS-case). The IRB assumption leads to a contradiction. Inflationary behavior on the visible brane can only be obtained if there is some contribution from the hidden brane. This is similar to the discussion of "remote" inflation in the RS-case. But here we have no choice: some "remote" contribution is required (in contrast to the RS-case where such a contribution was optional). The origin of this particular behavior is the presence of the dilaton as an additional bulk degree of freedom.

On the other hand, the presence of this additional bulk field opens the possibility to stabilize the radius with the dilaton field without the use of new external degrees of freedom. This situation is examined in section 4.4. We again have to do a perturbative expansion in $H^{2}$ (completed with a full numerical solution). Surprisingly this situation allows the conventional inflationary scenario where inflation is driven from the visible brane (with no "remote" contribution needed). The tensor modes can be computed and are found to be suppressed compared to the four-dimensional case (in contrast to the RS case). We also compare these results with the analysis of ref. [23] done in a conceptually different setup. Section 5 is devoted to conclusions and outlook.

\section{General considerations}

\subsection{Metric and expansion law}

We are interested in five-dimensional "braneworld" scenarios where gravity is propagating in five dimensions, while the Standard Model is confined to a four-dimensional slice of spacetime. The weak scale hierarchy problem can then be solved by an apparent, large 
four-dimensional Planck mass which is caused by the tiny overlap of the (massless) graviton zero mode with the visible brane.

In a very general manner, the action is given by

$$
S=\int \mathrm{d}^{4} x \mathrm{~d} z \sqrt{|g|}\left\{-\frac{M^{3}}{2} \mathcal{R}+\mathcal{L}_{b}(x, z)+\frac{1}{\sqrt{\left|g_{55}\right|}}\left[\mathcal{L}_{0} \delta(z)+\mathcal{L}_{\pi} \delta(z-\pi R)\right]\right\} .
$$

For a realistic cosmology, including a flat four-dimensional space, the most general ansatz for the metric can be written as

$$
\mathrm{d} s^{2}=n(z, t)^{2} \mathrm{~d} t^{2}-A(z, t)^{2} \delta_{i j} \mathrm{~d} x^{i} \mathrm{~d} x^{j}-b(z, t)^{2} \mathrm{~d} z^{2} .
$$

In hindsight of the properties of our anticipated solutions, however, we choose a simpler form of the metric as ansatz:

$$
\mathrm{d} s^{2}=f(z)^{2}\left(\mathrm{~d} t^{2}-a(t)^{2} \delta_{i j} \mathrm{~d} x^{i} \mathrm{~d} x^{j}\right)-\mathrm{d} z^{2} .
$$

The assumptions which allow for a reduction of (2.2) to the simpler form (2.3) are

(i) $\dot{b}(z, t)=0$ (the size of the extra dimension is static), and

(ii) $A(z, t)=f(z) a(t)$ ( $A$ is a separable function).

The first assumption (i) is certainly fulfilled if there is a mechanism to stabilize the size of the extra dimension, for example via a stabilizing potential for the radion mode [24]. Alternatively, if cosmological constants are the only form of energy density on the branes, one can achieve a consistent solution for a static extra dimension also by fine-tuning of the brane energy densities (cf. e.g. [19-21]). In either case, a stabilization will involve a contribution to the (55) component of the energy-momentum tensor $T_{M N} .{ }^{2}$ The (55) component of the Einstein equations, thus, serves to determine the size of the 5th dimension independently of the details of the stabilization mechanism [25-27]. This means that, as long as the stabilization mechanism decouples from all other equations, we can simply put aside the (55) equation while assuming that the radius is stabilized at some value (see e.g. [1]). We will later see that this is not always the case, and we will then also take into account the (55) equation. The remaining choice $b(z)=1$ then corresponds to a choice of coordinate system.

One can show that under the assumption (i), point (ii) is fulfilled if and only if $(n / A)$ is independent of $z$. Considering matching conditions on the four-dimensional branes, this requires that $\mathcal{L}_{0, \pi}$ is time independent [28], i.e. the energy densities are dominated by cosmological constants. This is a good assumption here, because we are interested in inflationary solutions of the scale factor $a(t)$.

The Einstein's equations are of the form

$$
G_{M N}=\mathcal{R}_{M N}-\frac{\mathcal{R}}{2} g_{M N}=\kappa^{2} T_{M N},
$$

with $\kappa^{2} \equiv M^{-3}$. With the ansatz (2.3) the following features arise:

\footnotetext{
${ }^{2}$ Our conventions are: $M, N, \ldots=0,1,2,3,5 ; \mu, \nu, \ldots=0,1,2,3 ; i, j, \ldots=1,2,3 ;$ metric signature $(+1,-1,-1,-1,-1)$; dots and primes denote the derivatives with respect to $t$ or $z$, respectively.
} 
- The $(00)$ and $(i j)$ equations are degenerate.

- The (05) equation is automatically fulfilled. Note that in the more general ansatz (2.2), the (05) equation gives rise to the insight that

$$
\frac{\dot{A}(z, t)}{n(z, t)}=\alpha(t),
$$

is independent of $z$ [26]. Due to the assumption (ii) of our ansatz above, however, this relation is automatically fulfilled here.

The only relevant Einstein equation, hence, is the (00) component of (2.4) which is given by

$$
-3 f^{2}\left\{\frac{f^{\prime \prime}}{f}+\frac{f^{\prime 2}}{f^{2}}\right\}+3 \frac{\dot{a}^{2}}{a^{2}}=\kappa^{2} T_{00} .
$$

Assuming the bulk and brane Lagrangians to be time independent it readily follows from (2.6) that

$$
H:=\frac{\dot{a}}{a},
$$

is constant. Upon integration

$$
a(t)=\mathrm{e}^{H t},
$$

and we realize that $H$ corresponds to the physical expansion rate of a three-dimensional slice of space at the five dimensional point $z_{0}$ where $f\left(z_{0}\right)=1$. The proper physical Hubble rate at a different slice of four-dimensional space time, say at $z=z_{1}$, can be obtained from $H$ by a redefinition of the time coordinate $f^{2}\left(z_{1}\right) \mathrm{d} t^{2} \rightarrow \mathrm{d} \tau^{2}$, and, therefore, is given by

$$
H_{z_{1}}=\frac{H}{f\left(z_{1}\right)} .
$$

\subsection{Effective Planck mass during inflation}

The relevant quantity for the actual strength of $4 \mathrm{D}$ gravity is the prefactor of the fourdimensional Ricci scalar (the normalization factor of the zero-mode graviton) which arises upon integrating out the 5 th dimension in (2.1). The effective $4 \mathrm{D}$ Planck mass $M_{\mathrm{Pl} \text {,eff }}$ obtained in this way is given by

$$
M_{\mathrm{Pl}, \mathrm{eff}}^{2}=M^{3} \int_{5 \mathrm{D}} \mathrm{d} z f(z)^{2} .
$$

Alternatively, one can deduce a four-dimensional Planck mass $M_{\mathrm{Pl} \text {,exp }}$ from the expansion law experienced on the visible brane

$$
H^{2}=\frac{\rho}{3 M_{\mathrm{Pl}, \exp }^{2}} .
$$

Here $\rho$ is the approximately constant energy density that drives inflation. In the picture of the IRB assumption, this energy density is due to a (sufficiently flat) inflaton potential on the visible brane.

The crucial point is that $M_{\mathrm{Pl} \text {,eff }}$ and $M_{\mathrm{Pl} \text {,exp }}$ will, in general, not coincide during inflation, thus, giving rise to a change of the tensor mode power spectrum as compared to inflation in the purely four dimensional case [1]. 


\subsection{Tensor modes in braneworld inflation}

As gravitational tensor modes are intrinsically bulk degrees of freedom, they are susceptible to the five dimensional geometry during inflation. For the treatment of tensor mode perturbations (primordial gravitational waves) we follow [1] (see also [2, 3]). Even though the discussion about the tensor modes in [1] is based on the IRB assumption that the inflaton field is confined to the visible brane, we remark that the result is applicable to general braneworld inflation including "remote inflation" where inflation is driven by an inflaton field located at the hidden brane. This is because the form of the zero mode graviton solution is independent of the specific dynamics responsible for inflation. How the spectrum of gravitational waves is modified then depends only on the underlying geometry and not on the microscopic details of the four-dimensional model of inflation.

The power spectrum of primordial tensor modes

$$
\mathcal{P}_{\mathrm{T}}(\ell)=\frac{2}{\pi^{2}}\left(\frac{H(\ell)}{M_{\mathrm{Pl}, \mathrm{eff}}}\right)^{2},
$$

generally deviates from its four-dimensional value. This can be attributed to a change of the effective reduced Planck mass $M_{\mathrm{Pl} \text {,eff }}$ during inflation [1]. Kaluza-Klein modes other than the massless tensor mode are not relevant because they are separated by a sufficiently broad mass gap [3].

In sharp contrast, scalar mode (density) perturbations originating from quantum fluctuations of the inflaton field do depend on the specific scenario of braneworld inflation. If one adopts the IRB assumption, the scalar perturbation is a purely four-dimensional degree of freedom confined to the visible brane. Then, given the ordinary four-dimensional Hubble law (2.11) on the brane, the presence of extra dimensions does (to leading order in slow-roll) not affect the power spectrum of scalar metric perturbations which is given by [1]

$$
\mathcal{P}_{\mathrm{S}}(\ell)=\frac{1}{8 \pi^{2}} \frac{1}{\epsilon}\left(\frac{H(\ell)}{M_{\mathrm{Pl}, \exp }}\right)^{2} .
$$

Consequently, the tensor-to-scalar ratio in scenarios with an extra dimension is modified to

$$
\frac{\mathcal{P}_{\mathrm{T}}(\ell)}{\mathcal{P}_{\mathrm{S}}(\ell)} \approx \frac{M_{\mathrm{Pl}, \mathrm{exp}}^{2}}{M_{\mathrm{Pl}, \mathrm{eff}}^{2}} \times\left.\frac{\mathcal{P}_{\mathrm{T}}(\ell)}{\mathcal{P}_{\mathrm{S}}(\ell)}\right|_{4 \mathrm{D}},
$$

if the IRB assumption is adopted. For other cases including "remote inflation", we could not make a definite statement on the tensor-to-scalar ratio at the moment. However, we still predict the altered behavior of primordial tensor modes based on the knowledge of the effective Planck mass during inflation.

\section{Large extra dimensions and Randall-Sundrum scenario}

\subsection{General form of the metric warping}

The actions of the LED model [5,6] and of the Randall-Sundrum model [7] are given by simplifications of (2.1). Let us consider the case of a bulk cosmological constant in addition 
to the unspecified radion stabilization mechanism in the bulk $\mathcal{L}_{b}(x, z)=-\Lambda+\mathcal{L}_{\operatorname{Rad}}(z)$. Then, the (00) Einstein equation (2.6) which determines the metric warping $f$ is given by

$$
\left(f^{2}\right)^{\prime \prime}-2 \frac{\dot{a}^{2}}{a^{2}}+\frac{2}{3} \kappa^{2} \Lambda f^{2}=0,
$$

locally in the bulk. Using the definition of $H$ as well as

$$
\mu^{2}:=-\frac{2}{3} \kappa^{2} \Lambda
$$

the (00) equation in the bulk reads

$$
\left(f^{2}\right)^{\prime \prime}-2 H^{2}-\mu^{2} f^{2}=0 .
$$

Depending on the sign of $\Lambda$, this equation has the general solutions (cf. also [29])

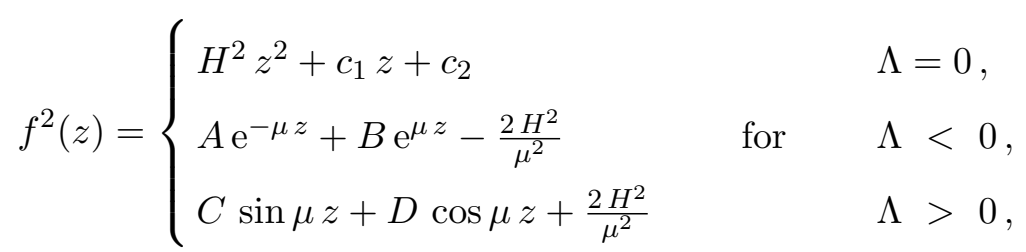

where we take $\mu>0$ without loss of generality. Each solution has two constants which are to be determined from the boundary conditions. Depending on the setting, the boundary conditions are set by symmetry constraints or the placement of branes. For example, if there is an infinitely thin brane with constant energy density ("brane tension") at a position $z=z_{0}$, the four-dimensional "brane" Lagrangian takes the form

$$
\mathcal{L}_{z_{0}}(x) \delta\left(z_{0}\right)=-\rho_{z_{0}} \delta\left(z_{0}\right) .
$$

This gives rise to a discontinuity of the first derivative of $f$ across the brane [30, 31] which is given by

$$
\frac{f^{\prime}\left(z_{0}^{+}\right)-f^{\prime}\left(z_{0}^{-}\right)}{f\left(z_{0}\right)}=-\frac{\kappa^{2}}{3} \rho_{z_{0}} .
$$

Solutions for $f$ in regions which are separated by branes are a priori unrelated. Relations between solutions for $f$ in the different regions can be obtained, for example, by requiring that the functions are related by the orbifold transformation $z \rightarrow-z$, or similar relations.

\subsection{Solution for the metric during inflation}

\subsubsection{Large extra dimensions}

The LED case is characterized by $\Lambda=0$ and the introduction of a single brane. The extra dimension is taken to be compact, with a size denoted by $2 \pi R$. We follow [1] and place the visible brane with tension $\rho_{0}=\rho$ at $z=z_{0}=0$. The boundary condition $f(0)=f(2 \pi R)$ fixes $c_{1}$, while the jump condition for the derivatives (3.6) evaluated at $z_{0}^{+}=0$ and $z_{0}^{-}=2 \pi R$ fixes the relation

$$
c_{2}=H^{2} \frac{6 \pi R}{\kappa^{2} \rho} .
$$


Recall that $H$ corresponds to the usual four-dimensional Hubble rate at the slice where $f(z)=1$. Since we are interested in the visible brane at $z=0$ we chose the four-dimensional coordinates such as to normalize $f(0)=1$ corresponding to $c_{2}=1$.

Altogether, the solution then is given by

$$
f^{2}(z)=H^{2}\left(z^{2}-2 \pi R z\right)+1, \quad \text { with } \quad H^{2}=\frac{\kappa^{2} \rho}{6 \pi R}
$$

Finally we can obtain the effective Planck mass in 4D from the metric warping and compare it to the effective Planck mass appearing in the expansion law. From the metric warping one finds

$$
M_{\mathrm{Pl}, \mathrm{eff}}^{2}=M^{3} \int_{0}^{2 \pi R} \mathrm{~d} z f(z)^{2}=M^{3} 2 \pi R\left(1-\frac{2}{3} \pi^{2} R^{2} H^{2}\right) .
$$

This shows that there is an $H$ dependent correction to the perceived strength of gravity in four dimensions as compared to the static case.

In contrast, from the four-dimensional Hubble expansion law one finds

$$
H^{2}=\frac{\kappa^{2} \rho}{6 \pi R} \equiv \frac{\rho}{3 M_{\mathrm{Pl}, \exp }^{2}} \quad \Rightarrow \quad M_{\mathrm{Pl}, \exp }^{2}=M^{3} 2 \pi R=\left.M_{\mathrm{Pl}, \mathrm{eff}}^{2}\right|_{H=0} ^{\mathrm{LED}} .
$$

We see that the differently derived four-dimensional Planck masses deviate during inflation. While $M_{\mathrm{Pl} \text {,exp }}$ is the relevant scale for perturbations of the scalar mode, $M_{\mathrm{Pl} \text {,eff }}$ is the relevant scale for the tensor mode perturbations. Consequently the tensor-to-scalar ratio is modified due to transdimensional effects [1].

The fact that the effective Planck mass in (3.9) is reduced shows that gravity is stronger during inflation, i.e. the tensor-to-scalar ratio is enhanced. In fact, if the Hubble scale is too large the metric warp function (3.8) crosses zero at which point there appear curvature singularities in the bulk. ${ }^{3}$ This signals the breakdown of perturbative gravity. Avoiding this situation results in an upper bound

$$
\pi^{2} R^{2} H^{2}<1
$$

This result generalizes to $n$ compact extra dimensions in the form $c_{n} \pi R H<1$, with a factor $c_{n} \sim \mathcal{O}(1)$ that depends on the details of the compactification [1]. Taking all 5D scales to be $M \sim \mu \sim \Lambda \sim \mathrm{TeV}$ and requiring a successful solution to the hierarchy problem one finds $R \sim 10^{30 / n-19} \mathrm{~m}$ resulting in an approximate upper bound on $H<10^{-30 / n} \mathrm{TeV}$ corresponding to a maximal reheating temperature of $T_{\mathrm{RH}}<10^{21 / 2-15 / n} \mathrm{GeV}$. For the specific case of $n=2$ this corresponds to $H<10^{-12} \mathrm{GeV}$ and $T_{\mathrm{RH}}<10^{3} \mathrm{GeV}$.

Depending on the assumed microscopic model of inflation there will eventually be even stronger bounds imposed on the product $H R$ by the (non-)observation of CMB $B$-mode polarization.

\footnotetext{
${ }^{3}$ We thank the referee for drawing our attention to this point.
} 


\subsubsection{Randall-Sundrum scenarios}

The Randall-Sundrum scenario [7] is characterized by taking $\Lambda<0$ while introducing two branes. Without loss of generality, the branes are placed at $z=0$ and $z=\pi R$. The respective brane tensions are denoted by $\rho_{0}$ and $\rho_{\pi}$, respectively. We will solve for $f(z)$ in the region $0 \leq z \leq \pi R$, while the solution in the region $-\pi R \leq z \leq 0$ can be obtained by the orbifold transformation $z \rightarrow-z$.

The general solution for $f(z)^{2}$ in the bulk is given by (cf. (3.4))

$$
f^{2}(z)=A \mathrm{e}^{-\mu z}+B \mathrm{e}^{\mu z}-\frac{2 H^{2}}{\mu^{2}},
$$

with two constants $A$ and $B$ that will shortly be fixed by boundary conditions. The effective four-dimensional Planck mass can be computed in a general fashion,

$$
M_{\mathrm{Pl}, \mathrm{eff}}^{2}=2 M^{3} \int_{0}^{\pi R} \mathrm{~d} z f(z)^{2}=\frac{2 M^{3}}{\omega \mu}(1-\omega)\left[B+\omega A-\frac{\omega}{(1-\omega)} \frac{2 H^{2} \pi R}{\mu}\right],
$$

where we have introduced the warp factor of the static case

$$
\omega:=\mathrm{e}^{-\mu \pi R} .
$$

Let us compute $A, B$, and $H$ for general (time-independent) boundary conditions. The junction conditions (3.6) take the form

$$
\frac{f^{\prime}(0)}{f(0)}=-\frac{\kappa^{2}}{6} \rho_{0}, \quad \text { and } \quad \frac{f^{\prime}(\pi R)}{f(\pi R)}=\frac{\kappa^{2}}{6} \rho_{\pi} .
$$

It is useful to define the dimensionless quantities

$$
\lambda_{0}:=\frac{\kappa^{2} \rho_{0}}{3 \mu}=\frac{\rho_{0}}{\sqrt{-6 M^{3} \Lambda}}, \quad \text { and } \quad \lambda_{\pi}:=\frac{\kappa^{2} \rho_{\pi}}{3 \mu}=\frac{\rho_{\pi}}{\sqrt{-6 M^{3} \Lambda}} .
$$

Obviously, $\lambda_{0}$ and $\lambda_{\pi}$ compare the brane tensions to the bulk cosmological constant. In particular, we will shortly see that for $\lambda_{0}=-\lambda_{\pi}= \pm 1$ the originally considered static case [7] is obtained. Altogether the constraints from the boundary conditions can be written as

$$
\begin{aligned}
f_{0}^{2} & =A+B-\frac{2 H^{2}}{\mu^{2}}, & f_{\pi}^{2} & =A \omega+B \omega^{-1}-\frac{2 H^{2}}{\mu^{2}}, \\
\lambda_{0} f_{0}^{2} & =A-B, & -\lambda_{\pi} f_{\pi}^{2} & =A \omega-B \omega^{-1},
\end{aligned}
$$

where we use the abbreviations

$$
f_{0}:=f(0), \quad f_{\pi}:=f(\pi R) .
$$

The first line of (3.17) are simple identities, while the second line arises from the junction conditions (3.15). Taking $\mu, \lambda_{0}, \lambda_{\pi}, R$ together with a normalization condition for $f$ (e.g. $\left.f_{0}=1\right)$ as input, this can be viewed as a system of four equations with four unknowns $\left(A, B, H, f_{\pi}\right)$. In particular, the expansion rate $H$ is a function of the input parameters 
and completely determined by the requirement that the jumps in the derivative of $f(z)$ at $z=0$ and $z=\pi R$ are consistent with the imposed brane tensions.

Let us take the visible brane to be located at $z=0$ and, therefore, adopt the normalization $f_{0}=1$. A general expression for the Hubble rate (at $z=0$ ) then is given by

$$
H^{2}=\frac{\mu^{2}}{2} \frac{\left(1-\lambda_{0}\right)\left(1-\lambda_{\pi}\right)-\omega^{2}\left(1+\lambda_{0}\right)\left(1+\lambda_{\pi}\right)}{\lambda_{\pi}(1-\omega)^{2}+\omega^{2}-1},
$$

while the parameters $A$ and $B$ can be expressed as

$$
A=\frac{1}{2}\left(1+\lambda_{0}\right)+\frac{H^{2}}{\mu^{2}}, \quad \text { and } \quad B=\frac{1}{2}\left(1-\lambda_{0}\right)+\frac{H^{2}}{\mu^{2}} .
$$

Let us reproduce some known results, while pointing out novel insights.

Static RS1. For an arbitrary but fixed radius $R$ (i.e. a fixed warp factor $\omega$ ) we see that the expansion rate (3.19) vanishes if $\lambda_{0}=-\lambda_{\pi}= \pm 1$. This corresponds to the cases ( $A=1, B=H=0)$ as well as $(A=H=0, B=1)$ implying that the metric takes the standard form

$$
\mathrm{d} s^{2}=\mathrm{e}^{\mp \mu z}\left(\mathrm{~d} t^{2}-\delta_{i j} \mathrm{~d} x^{i} \mathrm{~d} x^{j}\right)-\mathrm{d} z^{2} .
$$

Clearly, this is the originally considered RS1 case [7], which is static. For the case $\lambda_{0}=$ $-\lambda_{\pi}=-1$ we reproduce the well-known result

$$
\left.M_{\mathrm{Pl}, \mathrm{eff}}^{2}\right|_{H=0} ^{\mathrm{RS} 1}=\frac{2 M^{3}}{\omega \mu}(1-\omega)=\frac{2 M^{3}}{\mu}\left(\mathrm{e}^{\mu \pi R}-1\right),
$$

finding that $M_{\mathrm{Pl} \text {,eff }}$ appears exponentially enhanced over the fundamental scale $M$.

Note that tuning $\lambda_{0}$ and $\lambda_{\pi}$ to equal but opposite values \pm 1 is not the only possibility in order to obtain a static $(H=0)$ case. Alternatively, there can be a non-trivial interplay of the size of the fifth dimension and the expansion rate of the four-dimensional slices. For a large number of combinations of $\lambda_{0}$ and $\lambda_{\pi}$, see figure 1 , it is possible to tune $R$ to the very specific value

$$
R_{\mathrm{RS}}=\frac{1}{2 \mu \pi} \ln \left[\frac{\left(\lambda_{0}+1\right)\left(\lambda_{\pi}+1\right)}{\left(\lambda_{0}-1\right)\left(\lambda_{\pi}-1\right)}\right],
$$

which gives rise to a vanishing expansion rate of all four-dimensional slices. Most notably, for any given non-trivial value of $\lambda_{0} \neq \pm 1$ and any value of the radius one can always tune the tension of the other brane such as to stop inflation.

This clearly demonstrates that for inflationary solutions the evolution of the IR and UV branes are highly interdependent. In the most extreme case, for example, one could drive inflation of the visible brane by dynamics located solely on the hidden brane ("remote" inflation).

Expanding RS1. Using the general results (3.19) and (3.20), we can also reproduce the inflationary case considered by Giudice et al. [1]. Therefore, we take $\lambda_{0}=-1+\epsilon_{0}$ and $\lambda_{\pi}=1$. This corresponds to the usual fine tuned brane tensions of the static case plus an extra energy density $\rho_{0}$ on the visible brane, characterized by the dimensionless parameter

$$
\epsilon_{0}:=\frac{\kappa^{2} \rho_{0}}{3 \mu}
$$




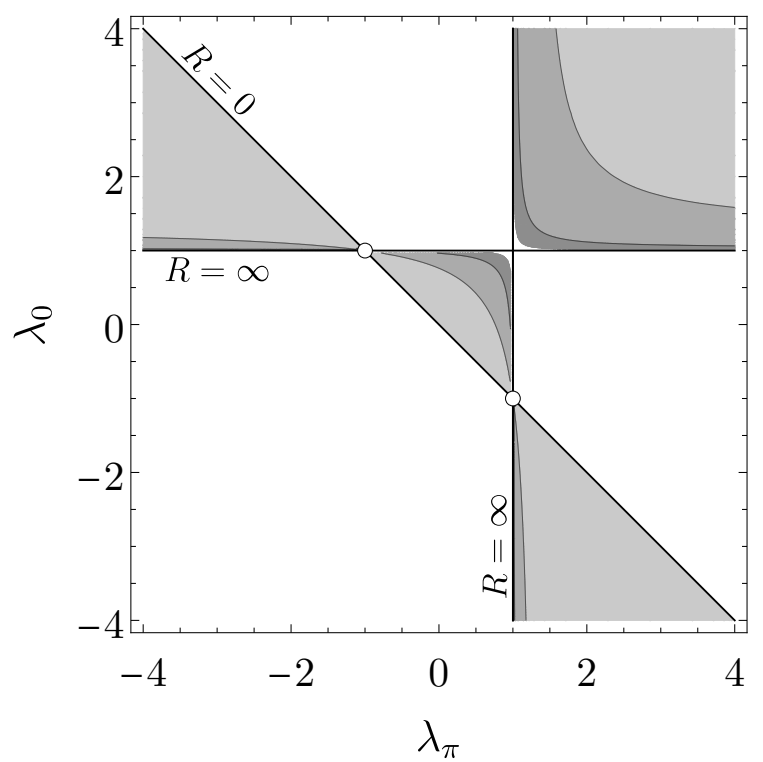

Figure 1. Allowed regions (filled) for the normalized brane tensions $\lambda_{0}, \lambda_{\pi}$. The contours illustrate the value of $R=R_{\mathrm{RS}}$ (darker means bigger) such that $H=0$. At the solid boundary lines $R$ asymptotes the values indicated in the plot. Values $\mu \pi R_{\mathrm{RS}} \approx 70$, for which the desired hierarchy between $M_{\mathrm{Pl} \text {,eff }}$ and $M$ is obtained, lie very close to the vertical and horizontal boundary lines. Empty circles show the choice of brane tensions in the original Randall-Sundrum scenario, where $H=0$ while $R$ at these points can take any value.

The Hubble rate comes out as

$$
H^{2}=\frac{\epsilon_{0} \mu^{2}}{2} \frac{\omega}{1-\omega}=\frac{\kappa^{2} \mu \rho_{0}}{6} \frac{\omega}{1-\omega}=\frac{\rho_{0}}{\left.3 M_{\mathrm{Pl}, \mathrm{eff}}^{2}\right|_{H=0} ^{\mathrm{RS} 1}},
$$

and the metric warping is given by

$$
f^{2}(z)=\frac{H^{2}}{\mu^{2} \omega} \mathrm{e}^{-\mu z}+\left[1+\frac{H^{2}}{\mu^{2}}\left(\frac{2 \omega-1}{\omega}\right)\right] \mathrm{e}^{\mu z}-\frac{2 H^{2}}{\mu^{2}} .
$$

The effective Planck mass during inflation then is

$$
M_{\mathrm{Pl}, \mathrm{eff}}^{2}=\left.M_{\mathrm{Pl}, \mathrm{eff}}^{2}\right|_{H=0} ^{\mathrm{RS} 1} \times\left[1-\frac{H^{2}}{\mu^{2}}\left(\frac{1}{\omega}-3+\frac{\omega}{1-\omega} 2 \pi R \mu\right)\right] .
$$

This result is exact. As shown in appendix A, our results for the expansion rate, metric warping and Planck mass agree with the results of [1] after taking into account the different conventions.

Clearly, the Planck mass is reduced during inflation, implying that the strength of gravity is enhanced. If the Hubble rate becomes too large, $f^{2}(z)$ crosses zero and there appears a curvature singularity in the bulk. Avoiding the onset of strongly coupled gravity thus imposes an upper bound on the Hubble rate

$$
H^{2}<\mu^{2} \frac{\omega}{(1-\omega)^{2}} \approx \mu^{2} \omega
$$


which also ensures that $M_{\mathrm{Pl} \text {,eff }}^{2}>0$. Taking all 5D scales to be $M \sim \mu \sim \Lambda \sim \mathrm{TeV}$ and requiring a solution to the hierarchy problem due to a warping $\mu \pi R \sim 70$ this bound restricts the Hubble rate to $H<10^{-12} \mathrm{GeV}$. Assuming maximally efficient reheating, this corresponds to a bound $T_{\mathrm{RH}}<10^{3} \mathrm{GeV}$. The fact that gravity is stronger during inflation generically leads to an enhancement of tensor mode perturbations.

As an interesting alternative, note that one could, in principle, also drive inflation from a completely remote sector that gives rise to an approximately constant additional energy density $\rho_{\pi}$ on the hidden brane. To model this we take $\lambda_{0}=-1, \lambda_{\pi}=1+\epsilon_{\pi}$, where

$$
\epsilon_{\pi}:=\frac{\kappa^{2} \rho_{\pi}}{3 \mu} .
$$

The Hubble rate of the visible brane comes out as

$$
H^{2}=\frac{\mu^{2} \epsilon_{\pi}}{2 \omega(1-\omega)-\epsilon_{\pi}(1-\omega)^{2}},
$$

and we have to restrict $\epsilon_{\pi}<2 \omega \ll 1$ to ensure that $H^{2}>0$. In the case $\epsilon_{\pi} \ll \omega$ one finds.

$$
H^{2} \approx \frac{\mu^{2} \epsilon_{\pi}}{2 \omega} \approx \frac{1}{\omega^{2}} \frac{\rho_{\pi}}{\left.3 M_{\mathrm{Pl}, \mathrm{eff}}^{2}\right|_{H=0} ^{\mathrm{RS} 1}} .
$$

This shows that the physical Hubble rate of the visible brane is highly susceptible to even smallest energy densities on the hidden brane. For example, the currently observed Hubble rate of $10^{-32} \mathrm{eV}$ on the visible brane can be caused by an additional energy density of only $10^{-70} \mathrm{eV}^{4}$ on the hidden brane. The necessary fine-tuning of energy density on the hidden brane demonstrates that the cosmological constant problem of our visible brane is not a local but in fact a global fine-tuning problem. Nevertheless, the necessary degree of finetuning on the hidden brane is the same as the usual $4 \mathrm{D}$ cosmological constant problem, as the natural mass scale on the hidden brane (for canonically normalized fields) is given by $\rho_{\pi} / \omega^{2}$.

The effective Planck mass during such a "remote" inflation caused by hidden-brane dynamics is given by

$$
M_{\mathrm{Pl}, \mathrm{eff}}^{2}=\left.M_{\mathrm{Pl}, \mathrm{eff}}^{2}\right|_{H=0} ^{\mathrm{RS} 1} \times\left[1+\frac{H^{2}}{\mu^{2}}\left(1+\omega-\frac{\omega}{1-\omega} 2 \pi R \mu\right)\right] .
$$

For this scenario the Planck mass is enhanced during inflation, implying that the strength of gravity is reduced. Gravity is weakly coupled throughout, meaning that there are no constraints on the possible values of $H$.

Despite the fact that the expansion law looks standard in terms of the canonical hiddenbrane energy density, we stress that it may not be possible here to directly interpret the effect of the altered effective Planck mass on the tensor-to-scalar ratio. In particular, inflation is driven by an energy density located on the hidden brane which sharply contradicts the IRB assumption that the inflaton dynamics should be confined to the visible brane. The results of [1] do not simply generalize to cases that violate this assumption. A dedicated study would be required to see how density and tensor mode perturbations on the visible brane can be affected or even seeded in other cases. Given that there is no direct 
coupling of the inflaton sector to the visible sector, reheating could occur via gravitational particle production [32] (cf. also [33-35]). The low efficiency of this reheating mechanism requires the inflation scale to be rather high, definitely well above the BBN scale. This is no problem here because $H$ is not bounded as discussed above. Exploring the observational consequences of such a "remote" inflation scenario is beyond the scope of this work.

Nihei-Kaloper-Kim-Kim special case. So far, we have not specified the mechanism which stabilizes the size of the fifth dimension. One possibility to obtain a fixed size of the extra dimension $R$ without any bulk dynamics is by fine-tuning the brane tensions against each other [19-21]. This is a very specific variant of the expanding RS1 case, in the sense that the four-dimensional slices may expand but the UV and IR brane tensions are fixed relative to each other in order to warrant that the fifth dimension is static.

With an empty bulk, the general solutions (3.4) receive an additional constraint from the (55) Einstein equation, which is then given by

$$
4 f^{\prime 2}-4 H^{2}-\mu^{2} f^{2}=0 .
$$

This restricts the coefficients of the general solution to the form

$$
A=\frac{H^{2}}{\mu^{2}} \widetilde{c_{0}}, \quad \text { and } \quad B=\frac{H^{2}}{\mu^{2}} \frac{1}{\widetilde{c_{0}}} \text {. }
$$

Using the parametrization $\widetilde{c_{0}} \equiv \mathrm{e}^{2 c_{0}}$ the metric warping results as

$$
f^{2}(z)=\frac{4 H^{2}}{\mu^{2}} \sinh ^{2}\left(-\frac{\mu}{2} z+c_{0}\right) .
$$

This is in full agreement with [21]. Due to the additional constraint, there is one less parameter than in the general solution. Furthermore, normalizing $f(0)=1$ fixes

$$
c_{0}=\operatorname{arcsinh}\left( \pm \frac{\mu}{2 H}\right)
$$

and there is no free parameter left.

The novel constraint is also manifest in the boundary conditions (3.15) which take the form

$$
\begin{aligned}
& \lambda_{0}=\operatorname{coth} c_{0}= \pm \sqrt{1+\frac{4 H^{2}}{\mu^{2}}}, \\
& \lambda_{\pi}=-\operatorname{coth}\left(-\frac{\mu \pi R}{2}+c_{0}\right) .
\end{aligned}
$$

It immediately follows from eqs. (3.37) that $\left|\lambda_{0, \pi}\right|>1$ and there is a relation between the size $R$ of the fifth dimension and the brane tensions ${ }^{4}$

$$
R=R_{1} \equiv \frac{1}{\mu \pi} \ln \left[\frac{\left(\lambda_{0}+1\right)\left(\lambda_{\pi}+1\right)}{\left(\lambda_{0}-1\right)\left(\lambda_{\pi}-1\right)}\right] .
$$

The metric warping together with the boundary condition $\lambda_{\pi}$ as a function of the chosen radius for a fixed value of $\lambda_{0}$ is displayed in figure 2. Furthermore, the Hubble rate of the

\footnotetext{
${ }^{4}$ As a curiosity, note that this is precisely $2 R_{\mathrm{RS}}$ (3.23).
} 


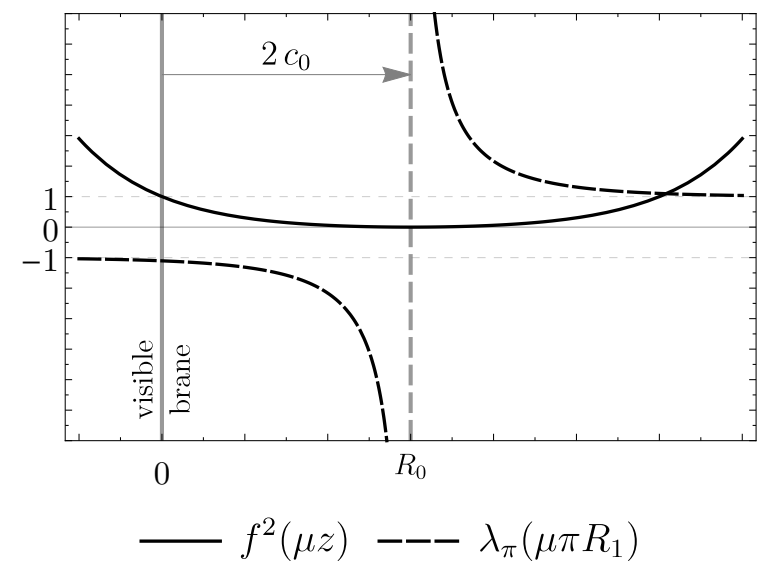

Figure 2. The metric warping (3.35) of the Nihei-Kaloper-Kim-Kim special case (solid) normalized such that $f(0)=1$. The dashed line shows the normalized brane tension $\lambda_{\pi}\left(R_{1}\right)$ which the hidden brane must carry if it would be located at a distance $R_{1}$ away from the visible brane. There is a special radius $z=\pi R_{0}$ for which the metric is zero and $f^{\prime} / f$ does not exist. If a brane is placed at this special radius it decouples and can have any brane tension.

visible brane (as always in our convention at $z=0$ ) comes out as

$$
H^{2}=\frac{\mu^{2}}{4}\left(\lambda_{0}^{2}-1\right)
$$

while the Hubble rate at the hidden brane (located at $z=\pi R_{1}$ ) is given by

$$
H_{\pi}^{2} \equiv \frac{H^{2}}{f_{\pi}^{2}}=\frac{\mu^{2}}{4}\left(\lambda_{\pi}^{2}-1\right)
$$

Our solutions fully agree with eqs. (16) and (20) of [21] after noting that their $k \equiv \mu / 2$, $L_{5} \equiv \pi R$, and $k_{1,2} / k \equiv \lambda_{0, \pi}$. However, the discussion in [21] was limited to the parameter region $1<\lambda_{0}<-\lambda_{\pi}$. This limitation makes sense if the hierarchy problem is to be solved at the $z=\pi R_{1}$ brane and if one requires that the metric should not have a zero at any point in the extra dimension. On the other hand, if we do not impose these requirements we find that there are additional regions in parameter space for which a consistent solution can be found, cf. figure 3 (see also [19, 20]). In particular, we find that there is another solution for the size of the extra dimension

$$
R=R_{0} \equiv \frac{1}{\mu \pi} \ln \left[\frac{\left(\lambda_{0}+1\right)}{\left(\lambda_{0}-1\right)}\right]
$$

for which the general solution for $H^{2}$ (3.19) is reconciled with (3.39). This solution makes sense when $\lambda_{0}>1$ (i.e. $c_{0}>0$ ), irrespective of the value of $\lambda_{\pi}$. For this solution, the fine-tuned value of the radius is independent of the energy density on the hidden brane! In order to understand why this is the case, it is important to note that $z=\pi R_{0}$ corresponds to the zero of the metric (3.35) (cf. figure 2). Despite the fact that $f^{\prime}\left(\pi R_{0}\right) / f\left(\pi R_{0}\right)$ does not exist, this second solution for the radius is consistent with the boundary conditions for 


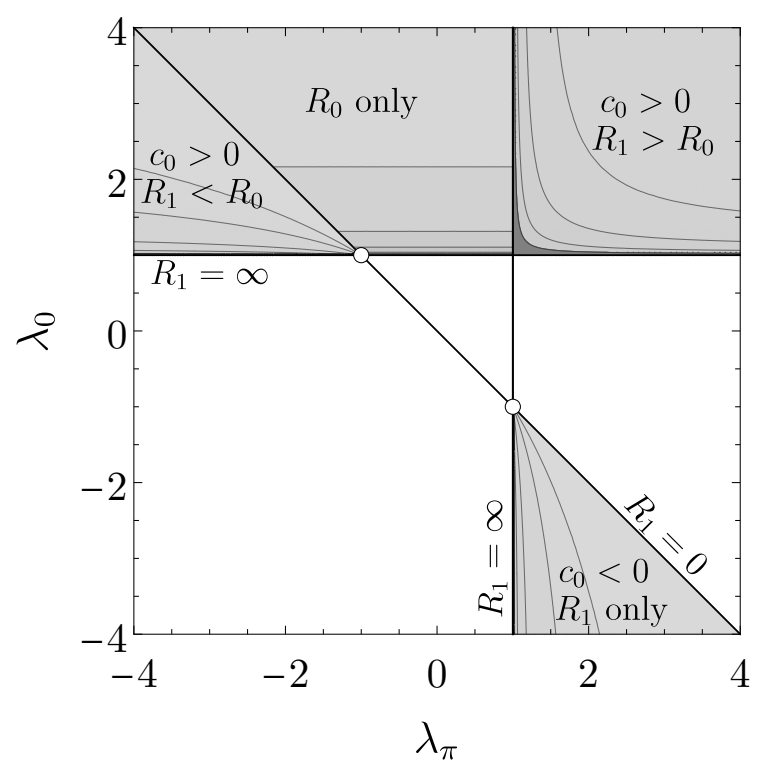

Figure 3. Allowed regions (filled) for the normalized brane tensions $\lambda_{0}, \lambda_{\pi}$ for the Nihei-KaloperKim-Kim special case. The contours illustrate the value of $R_{1}$ where it is allowed and otherwise the value of $R_{0}$ (darker means bigger) such that a consistent solution is achieved. The Hubble rate of the visible brane is everywhere given by (3.39). Contour lines with constant $R$ can also be viewed as parametric curves on which the general expression for $H^{2}$, Equation (3.19), is reconciled with (3.39).

any value of $\lambda_{\pi}$. This may appear surprising in view of the condition (3.37). However we note that in deriving (3.6), which is the general origin of (3.37), it was tacitly assumed that the metric has no zero $f\left(z_{0}\right) \neq 0$. Without imposing this requirement the actual boundary conditions for a brane at $z=z_{0}$ read

$$
-3 f\left(z_{0}\right)\left[f^{\prime}\left(z_{0}^{+}\right)-f^{\prime}\left(z_{0}^{-}\right)\right]=f\left(z_{0}\right)^{2} \kappa^{2} \rho_{z_{0}} .
$$

It follows that at metric zeros $f\left(z_{0}\right)=0$ the boundary conditions are trivially fulfilled and the parameter $\rho_{z_{0}}$ will not enter the solution. The expansion rate of the hidden brane, $H_{z_{0}}$, becomes meaningless in this case. We conclude that putting the hidden brane precisely at the zero of the metric is a valid solution for which the hidden brane decouples and does not play any role for physics on the visible brane.

Let us discuss how the hierarchy problem can be addressed in the NKKK setup, while pointing out the effect on the inflationary tensor modes. The (orbifolded) extra dimension extends between the visible brane at $z=0$ and a hidden brane at $z=\pi R_{1}$ with the general solution for the metric given by (3.35). Integrating out the extra dimension, we find the strength of effective four-dimensional gravity to be

$$
M_{\mathrm{Pl}, \mathrm{eff}}^{2}=\left.M_{\mathrm{Pl}, \mathrm{eff}}^{2}\right|_{H=0} ^{\mathrm{RS} 1} \times \frac{H^{2}}{\mu^{2}}\left[\mathrm{e}^{ \pm 2 \operatorname{arcsinh} \frac{\mu}{2 H}}+\omega \mathrm{e}^{\mp 2 \operatorname{arcsinh} \frac{\mu}{2 H}}-\frac{\omega}{1-\omega} 2 \pi R \mu\right] .
$$

The two signs correspond to the two cases $c_{0} \lessgtr 0$. There are no restrictions on the possible values of $H$ and $R$ from this since $M_{\mathrm{Pl} \text {,eff }}^{2}$ is strictly positive. In all cases where the hierarchy 
problem is solved, the Planck mass is enhanced during inflation implying that gravity is weakened and the tensor mode perturbations are reduced. For $c_{0} \lessgtr 0$ and taking the limit $H \rightarrow 0$ (corresponding to $\left|\lambda_{0}\right| \rightarrow 1$ ) the metric approaches the standard RS warped form $f(z)^{2}=\mathrm{e}^{ \pm \mu z}$ with an effective Planck mass

$$
M_{\mathrm{Pl}, \mathrm{eff}}^{2} \stackrel{H \rightarrow 0}{\longrightarrow} \begin{cases}\left.M_{\mathrm{Pl}, \mathrm{eff}}^{2}\right|_{H=0} ^{\mathrm{RS} 1}, & \text { for } c_{0}<0, \\ \left.M_{\mathrm{Pl}, \mathrm{eff}}^{2}\right|_{H=0} ^{\mathrm{RS} 2} \equiv \frac{2 M^{3}}{\mu}(1-\omega), & \text { for } c_{0}>0 .\end{cases}
$$

The hierarchy problem at the visible brane (without loss of generality placed at $z=0$ ) can be reliably addressed only in the case $c_{0}<0$ where the metric warping increases away from the brane.

In the case $c_{0}>0$ and $R_{1}>R_{0}$ the zero of $f(z)^{2}$ is present in the bulk. In contrast to the cases discussed in the previous sections, the zero of $f(z)^{2}$ does not correspond to a true (e.g. curvature) singularity but is instead related to the presence of a causal horizon in the extra dimension $[2,20]$. Following, for example, $[2,36]$ one can restrict the size of the extra dimension to a causally connected region, i.e. let the extra dimension end on the horizon at $\pi R_{0}=2 c_{0} / \mu$. In this case the integral (2.10) results in

$$
M_{\mathrm{Pl}, \mathrm{eff}}^{2}=\frac{2 M^{3}}{\mu} \frac{2 H^{2}}{\mu^{2}}\left[\sinh \left(2 \operatorname{arcsinh} \frac{\mu}{2 H}\right)-2 \operatorname{arcsinh} \frac{\mu}{2 H}\right] .
$$

This case cannot address the hierarchy problem. The effective Planck mass only implicitly depends on the size of the extra dimension via $H$ which is in a one-to-one relationship with $R$. The extra dimension can become infinite in size only if $H \rightarrow 0$, in which case we approach the RS2 limit.

A naive second possibility to address the hierarchy problem would be $c_{0}>0$ with $R_{1}>R_{0}$, corresponding to the upper right region of figure 3 where $\lambda_{0}, \lambda_{\pi}>1$ and the metric is cusped upwards on both branes (cf. figure 2). However, in this case the causal horizon is present in the bulk and the extra dimension consists of two causally disconnected regions without interaction. The graviton zero mode then would have to be normalized in causally connected regions only, corresponding to the previously discussed case that the extra dimension ends on the horizon (3.45).

A different possibility could arise if the NKKK case is only the late time limit to a situation in which there was initially no horizon but it formed dynamically. Just before the time of horizon formation the graviton zero mode would have to be normalized to the full extra dimension and it remains to be investigated in a fully dynamical setting how this normalization would change after the horizon is formed. Exploring this possibility would require a fully dynamical treatment of the extra dimension and the formation of the horizon, which is beyond the scope of this work and so we will not further discuss this here.

Expanding RS2. Let us also discuss the RS2 model [22]. Here, the brane with positive tension is the visible brane centered at $z=0$, and the radius of the extra dimension is eventually taken to infinity. This model does not solve the hierarchy problem, but it is of 
interest simply due to the fact that an infinite extra dimension is allowed in consistency with observation.

The original (static) RS2 setup is obtained by choosing $\lambda_{0}=-\lambda_{\pi}=1$. In this case the effective four-dimensional Planck mass is

$$
\left.M_{\mathrm{Pl}, \mathrm{eff}}^{2}\right|_{H=0} ^{\mathrm{RS} 2}=\frac{2 M^{3}}{\mu}(1-\omega)=\frac{2 M^{3}}{\mu}\left(1-\mathrm{e}^{-\mu \pi R}\right) .
$$

There is no exponential hierarchy generated between the $5 \mathrm{D}$ and $4 \mathrm{D}$ scales, and there is no obstruction in taking $R \rightarrow \infty$.

For the expanding case, we assume that the inflaton sector is confined to the visible brane. Therefore, we keep $\lambda_{\pi}=-1$ but add a surplus energy density to the visible brane given by $\lambda_{0}=1+\epsilon_{0}$, with $\epsilon_{0}$ as given in (3.24). The general results (3.4) and (3.19) apply and one finds a Hubble rate

$$
H^{2}=\frac{\mu^{2} \epsilon_{0}}{2(1-\omega)}=\frac{\rho_{0}}{\left.3 M_{\mathrm{Pl}, \mathrm{eff}}^{2}\right|_{H=0} ^{\mathrm{RS} 2}},
$$

which is consistent with the usual four-dimensional expansion law.

The metric warping is given by

$$
f^{2}(z)=\left[1+\frac{H^{2}}{\mu^{2}}(2-\omega)\right] \mathrm{e}^{-\mu z}+\frac{H^{2}}{\mu^{2}} \omega \mathrm{e}^{\mu z}-\frac{2 H^{2}}{\mu^{2}},
$$

and the resulting effective Planck mass is

$$
M_{\mathrm{Pl}, \mathrm{eff}}^{2}=\left.M_{\mathrm{Pl}, \mathrm{eff}}^{2}\right|_{H=0} ^{\mathrm{RS} 2} \times\left[1-\frac{H^{2}}{\mu^{2}}\left(\omega-3+\frac{2 \mu \pi R}{1-\omega}\right)\right]
$$

The correction factor always reduces the effective Planck mass, i.e. it increases the strength of gravity during inflation. Since the discussion of [1] is fully applicable, we conclude that the tensor modes during inflation are enhanced in the RS2 setup.

Analogous to the LED and RS1 cases the metric can cross zero at which point a curvature singularity appears in the bulk. Avoiding this situation imposes the bound

$$
H^{2}<\mu^{2} \omega
$$

which also ensures $M_{\mathrm{Pl} \text {,eff }}^{2}>0$. Obeying this bound enforces $H \rightarrow 0$ as $R \rightarrow \infty$ implying that the inflating RS2 case is inconsistent with taking the size of the extra dimension to infinity. In principle this is nothing new, as it also happens in the LED case, cf. Equation (3.9) and the related discussion in [1]. The fact that we cannot take the size of the extra dimension to infinity without letting $H \rightarrow 0$ is independent of whether we take into account the (55) equation or not, as is clear from the previous section. This conclusion does not change in case one allows for other values of $\lambda_{\pi}$ (for example, $\lambda_{\pi}=0$ or $\lambda_{\pi}=-1+\epsilon_{0}$ ). 


\section{Linear dilaton model}

\subsection{Generalities}

Let us consider the linear dilaton configurations [8-11,37] of little string theory (see [38] for more references on LST). This case is akin to the RS case in the sense that there will be a negative bulk cosmological constant and two branes. The crucial difference to the RS case is the presence of an additional scalar field, the dilaton. Ultimately, this gives rise to power law warping in contrast to the exponential warping of the RS case.

The action in the Einstein frame is given by $[9,10,16,23]$

$$
\begin{aligned}
S=\int \mathrm{d}^{4} x \mathrm{~d} z \sqrt{|g|}\left\{\frac{M^{3}}{2}\right. & \left(-\mathcal{R}+\frac{1}{3} g^{M N} \partial_{M} S \partial_{N} S+4 k^{2} \mathrm{e}^{-\frac{2}{3} S}\right) \\
& \left.+\frac{\mathrm{e}^{-\frac{1}{3} S}}{\sqrt{\left|g_{55}\right|}}\left[\mathcal{L}_{0} \delta(z)+\mathcal{L}_{\pi} \delta(z-\pi R)\right]+\mathcal{L}_{b}(x, z)\right\},
\end{aligned}
$$

where $-2 k^{2} \equiv \kappa^{2} \Lambda$ and we have allowed for the possibility of having branes and extra contributions in the bulk. $S$ is the (dimensionless) dilaton and the corresponding canonically normalized field is $\phi=M^{3 / 2} S / \sqrt{3}$.

The dilaton field itself can be used to stabilize the radius of the fifth dimension. Alternatively, one could also introduce a Goldberger-Wise (GW) type scalar field [24] fulfilling the same purpose. In analogy to the LED and RS case, we will first be agnostic about the details of the radius stabilization and simply ignore the corresponding (55) Einstein equation, assuming that it gives rise to a stable radius. We are again looking for solutions to Einstein's equations that satisfy the metric ansatz

$$
\mathrm{d} s^{2}=f(z)^{2}\left(\mathrm{~d} t^{2}-a(t)^{2} \delta_{i j} \mathrm{~d} x^{i} \mathrm{~d} x^{j}\right)-\mathrm{d} z^{2} .
$$

The Einstein tensor is the same as above, while the energy-momentum tensor now is given by

$$
\kappa^{2} T_{M N}=\frac{1}{3} \partial_{M} S \partial_{N} S-\frac{1}{6} g^{P Q} \partial_{P} S \partial_{Q} S g_{M N}+\kappa^{2} \Lambda \mathrm{e}^{-2 S / 3} g_{M N} .
$$

The (00) equation in the bulk then can be written as

$$
\left(f^{2}\right)^{\prime \prime}-2 H^{2}+\frac{2}{3} \kappa^{2} \Lambda f^{2} \mathrm{e}^{-2 S / 3}+\frac{f^{2} S^{\prime 2}}{9}=0 .
$$

Here we are assuming that the dilaton is homogeneous in four dimensions $S=S(z)$. It follows that $H=$ const. and the $(i j)$ and (00) equations are degenerate. Additionally, there appears the equation of motion of the dilaton, which is in the bulk given by

$$
S^{\prime \prime}+4 \frac{f^{\prime}}{f} S^{\prime}+2 \kappa^{2} \Lambda \mathrm{e}^{-2 S / 3}=0 .
$$

The Bianchi identity is identical to the dilaton equation of motion and does not give an independent constraint.

Due to the exponential dilaton factors in the action, the boundary conditions are modified in comparison to the previous cases. For a brane at position $z=z_{0}$ carrying a 
constant four-dimensional energy density $\mathcal{L}_{z_{0}}=-\rho_{z_{0}}$ the discontinuities of $f^{\prime}$ and $S^{\prime}$ across the brane have to fulfill

$$
\frac{f^{\prime}\left(z_{0}^{+}\right)-f^{\prime}\left(z_{0}^{-}\right)}{f\left(z_{0}\right)}=-\frac{\kappa^{2} \rho_{z_{0}}}{3} \mathrm{e}^{-\frac{1}{3} S\left(z_{0}\right)}
$$

as well as

$$
S^{\prime}\left(z_{0}^{+}\right)-S^{\prime}\left(z_{0}^{-}\right)=-\kappa^{2} \rho_{z_{0}} \mathrm{e}^{-\frac{1}{3} S\left(z_{0}\right)}
$$

We were not able to find a closed form solution for (4.4) and (4.5) for a general $H \neq 0$. We will, thus, discuss the exactly solved static case $(H=0)$ first, after which we present a perturbative solution for the expanding case.

It should be mentioned here that a closed form solution for the LD model in the Jordan frame has been presented in [39]. Despite the difficulties in transforming the solution to the Einstein frame [39] their derivation makes use of the unperturbed (55) equation. This is why we cannot directly adopt their solution here. Nevertheless, let us emphasize that it would be eminently useful to have an exact solution also for the LD case, just as for the LED and RS cases above.

\subsection{The static case}

Setting $H=0$, the most general simultaneous solution to (4.4) and (4.5) in the bulk is given by

$$
S(z)=3 \ln \frac{f(z)}{c_{S}}, \quad f(z)=c_{0}+\zeta c_{S} \frac{2 k}{3} z .
$$

Here, $c_{0}$ and $c_{S}$ are arbitrary dimensionless constants and $\zeta= \pm 1$ is an undetermined sign. By rescaling of the four-dimensional coordinates it is always possible to normalize $f(0)=1$, thus, fixing the constant $c_{0}=1$. By contrast, $c_{S}$ corresponds to the normalization of $S(0)$, whose value, however, can also be chosen without loss of generality. This can be understood by noting that $c_{S}$ can be absorbed into $\tilde{k}:=c_{S} k$ and it thereby disappears completely from the bulk action which now contains $\tilde{k}$ and $\tilde{S}(z)=3 \ln (1+2 \tilde{k} z / 3)$ which is automatically normalized to $\tilde{S}(0)=0$. Reformulating the brane Lagrangians in terms of $\tilde{S}$ one finds that they have to be globally rescaled by $c_{S}$, for example $\mathcal{L}_{0} \rightarrow c_{S} \mathcal{L}_{0}$. To maintain canonically normalized kinetic terms on the branes one then has to rescale the fields in $\mathcal{L}_{0}$ which likewise leads to an unphysical rescaling of couplings. Thereby $c_{S}$ can be completely absorbed from the theory without loss of generality. Correspondingly, a normalization of $S(0)=0$ can always be chosen without physical consequences [16]. We stress this point here because in the expanding $(H \neq 0)$ case below this conclusion will not hold and the physical results change if the boundary condition $S(0)=0$ is changed. The bulk solution of the static case is consistent with the boundary conditions on the two branes (4.6), (4.7) only for the fine-tuned brane tensions ${ }^{5}$

$$
-\rho_{0}=\rho_{\pi}=\zeta 4 k M^{3} .
$$

\footnotetext{
${ }^{5}$ Note that this corresponds to values $-\lambda_{0}=\lambda_{\pi}=2 / \sqrt{3}$ in our above notation for the RS case, showing that the fine-tuned brane tensions in the $\mathrm{CW}$ case are different from the ones required in the RS case.
} 
Here, it makes sense to define the dimensionless quantities

$$
\eta_{0}:=\frac{\rho_{0}}{4 k M^{3}}, \quad \text { and } \quad \eta_{\pi}:=\frac{\rho_{\pi}}{4 k M^{3}} .
$$

Choosing $\zeta=+1$, i.e. $\eta_{0}=-\eta_{\pi}=-1$ and assuming the usual orbifold symmetry $z \rightarrow-z$ for solutions in the two separate domains, one obtains

$$
f(z)=f_{s}(z):=1+\frac{2 k}{3}|z| \quad \text { and } \quad S(z)=S_{s}(z):=3 \ln \left(1+\frac{2 k}{3}|z|\right) .
$$

This is the standard linear dilaton solution (see e.g. [9-11]) which appears here as "logarithmic dilaton" due to our euclidean coordinate choice for the fifth dimension. Compared to the exponential warping in the static RS metric (3.21) we find here a power-law warping. Taking the fifth dimension to be of size $z \in[-\pi R, \pi R]$ the effective four-dimensional Planck mass is given by

$$
\left.M_{\mathrm{Pl}, \mathrm{eff}}^{2}\right|_{H=0} ^{\mathrm{LD}}=2 M^{3} \int_{0}^{\pi R} \mathrm{~d} z f(z)^{2}=\frac{M^{3}}{k}\left[\left(1+\frac{2 k}{3} \pi R\right)^{3}-1\right] .
$$

Taking the fundamental scale to be $M \sim k \sim \mathrm{TeV}$ and requiring the observed value for $M_{\mathrm{Pl} \text {,eff }}$ we find that $k \pi R \gtrsim 10^{11}$, corresponding to an extra dimension of size $\sim 10 \mathrm{~nm}$.

Note that it is crucial to choose the same sign for $\zeta$ and the possible values of $z$. Choosing $\zeta=-1$ (or equivalently allowing for negative values of $z$ ) the metric vanishes and the dilaton profile diverges at $z_{\text {sing }}=(-) 3 / 2 k$ corresponding to a physical singularity. This would give rise to a natural cutoff size of the extra dimension $\pi R \leq 3 / 2 k$. The presence of such singularities has already been noted in [39]. We are interested in cases where the presence of the extra dimension solves the hierarchy problem. Therefore, we limit ourselves to parameters which allow for an arbitrary size of the extra dimension and avoid the singularity in the dilaton profile.

\subsection{The expanding case with external stabilization}

Let us now generalize the solution to the case $H \neq 0$. Since we were not able to find an exact solution for $f(z)$ and $S(z)$ for the general case, we will assume that the dimensionless quantity $\delta:=H^{2} / k^{2}$ is small and find a perturbative solution in $\delta$. In the limit $\delta \rightarrow 0$ the linear dilaton solution should be recovered. Therefore, we adopt the ansatz

$$
f(z)=f_{s}(z)[1+\delta d f(z)], \quad \text { and } \quad S(z)=S_{s}(z)+\delta d S(z),
$$

where $f_{s}(z)$ and $S_{s}(z)$ are the solutions of the static case given in (4.11). Plugging the ansatz into equations (4.4) and (4.5) we expand in $\delta \ll 1$ and use that $f_{s}$ and $S_{s}$ are solutions of the static case. At linear order in $\delta$ we find that $d f(z)$ and $d S(z)$ have to fulfill

$$
\begin{aligned}
2 f_{s}^{2} \frac{d f^{\prime \prime}}{k^{2}}+\frac{16}{3} f_{s} \frac{d f^{\prime}}{k}+\frac{4}{9} f_{s} \frac{d S^{\prime}}{k}+\frac{8}{9} d S-2 & =0 \\
f_{s} \frac{d S^{\prime \prime}}{k^{2}}+\frac{8}{3} \frac{d S^{\prime}}{k}+\frac{8}{3} \frac{d S}{f_{s}}+8 \frac{d f^{\prime}}{k} & =0 .
\end{aligned}
$$


These two equations can be decoupled, thereby giving rise to a third oder equation for $d S$ which can be solved. Subsequently the solution for $d S$ can be used in order to solve also for $d f$. The corrections to the bulk solutions are then given by

$$
\begin{aligned}
d S(z) & =-\frac{9}{2}+\frac{c_{1}}{\left[3 f_{s}(z)\right]^{3}}+\frac{c_{2}}{\left[3 f_{s}(z)\right]^{2}}+\frac{c_{3}}{3 f_{s}(z)}, \\
d f(z) & =\frac{9}{4} \ln f_{s}(z)+\frac{c_{1}}{162 f_{s}(z)^{3}}+\frac{c_{2}}{54 f_{s}(z)^{2}}+\frac{c_{3}}{9 f_{s}(z)}+c_{4},
\end{aligned}
$$

with four arbitrary constants $c_{1-4}$. Again, we normalize $f(0)=1$ and $S(0)=0$ by fixing $c_{4}$ as well as $c_{3}$, respectively.

The junction conditions on the branes are

$$
\begin{array}{ll}
\frac{f^{\prime}(0)}{f(0)}=-\frac{2}{3} k \eta_{0} \mathrm{e}^{-S(0) / 3}, & \frac{f^{\prime}(\pi R)}{f(\pi R)}=\frac{2}{3} k \eta_{\pi} \mathrm{e}^{-S(\pi R) / 3} \\
S^{\prime}(0)=-2 k \eta_{0} \mathrm{e}^{-S(0) / 3}, & S^{\prime}(\pi R)=2 k \eta_{\pi} \mathrm{e}^{-S(\pi R) / 3}
\end{array}
$$

As our bulk solution is only valid up to order $\delta$ we can only require that the boundary conditions are solved up to that order. This implies that deviations of the brane tensions should be small compared to the static case, i.e. $\eta_{0}=-1+\epsilon_{0}$ and $\eta_{\pi}=1+\epsilon_{\pi}$ with $\epsilon_{0}, \epsilon_{\pi} \ll 1$. The boundary conditions are then solved by

$$
\begin{array}{ll}
c_{1}=\frac{243}{2}\left(f_{s, \pi}^{2}+f_{s, \pi}\right), & c_{2}=-\frac{243}{4}\left(f_{s, \pi}^{2}+f_{s, \pi}+1\right), \\
c_{3}=\frac{27}{4}\left(f_{s, \pi}^{2}+f_{s, \pi}+5\right), &
\end{array}
$$

together with the relations

$$
\delta=\frac{4 \epsilon_{0}}{3\left(f_{s, \pi}^{2}+f_{s, \pi}-1\right)}, \quad \text { and } \quad \frac{\epsilon_{\pi}}{\epsilon_{0}}=\frac{f_{s, \pi}^{2}-f_{s, \pi}-1}{f_{s, \pi}^{2}\left(f_{s, \pi}^{2}+f_{s, \pi}-1\right)} .
$$

Here we have used the abbreviation $f_{s, \pi} \equiv f_{s}(\pi R)$. Finally, our solution for the metric warping is fully specified and given by

$$
f(z)=1+\frac{2 k}{3}|z|-H^{2} \pi^{2} R^{2} k|z| \frac{2\left(9+3 k|z|+2 k^{2} z^{2}\right)}{9(3+2 k|z|)^{2}}+\text { h.o. },
$$

where h.o. denotes terms of higher order in $H^{2} / k^{2} \ll 1$ or lower order in $k \pi R \gg 1$. For completeness, we also state the leading order correction to the dilaton profile which is given by

$$
S(z)=3 \ln \left(1+\frac{2 k}{3}|z|\right)+H^{2} \pi^{2} R^{2} k|z| \frac{6(2 k|z|-3)}{(2 k|z|+3)^{3}}+\text { h.o. . }
$$

Our perturbative solutions agree well with a numerical solution of (4.4) and (4.5), as displayed in figure 4 .

Given the metric warping $f(z)$, the effective Planck mass during inflation can be computed to be

$$
M_{\mathrm{Pl}, \mathrm{eff}}^{2}=\left.M_{\mathrm{Pl}, \mathrm{eff}}^{2}\right|_{H=0} ^{\mathrm{LD}} \times\left(1-\frac{1}{3} H^{2} \pi^{2} R^{2}+\text { h.o. }\right) \text {. }
$$




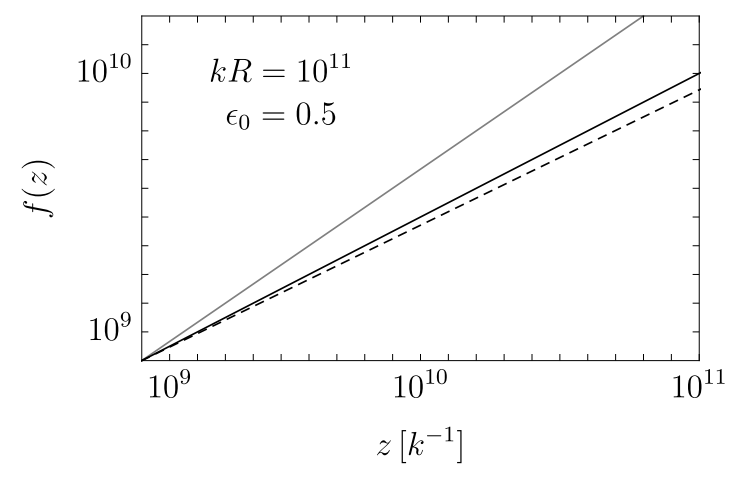

(a)

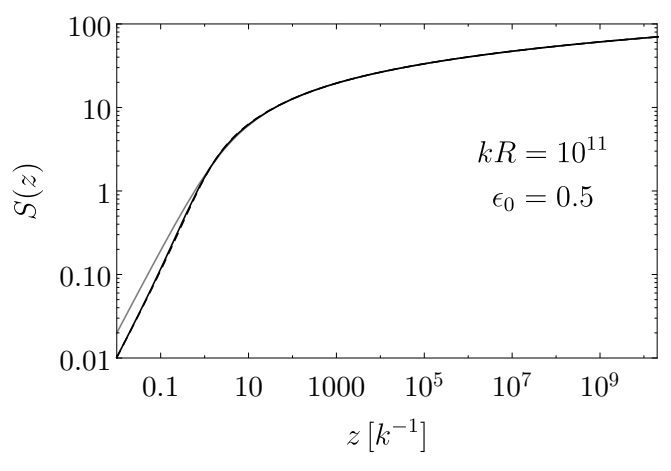

(b)

Figure 4. Metric warping (a) and Dilaton profile (b) of the Linear Dilaton model with inflation (black) as compared to the static solution (gray). Next to our perturbative results (black) we also show a numerical solution (black, dashed). For the chosen parameters, the resulting Hubble rate is $H \approx 4 \times 10^{-12} k \approx 4 \times 10^{-9} \mathrm{GeV}$.

The inferred bound on $H R$ from the perturbativity requirement on gravity $M_{\mathrm{Pl} \text {,eff }}^{2}>0$ during inflation is

$$
H^{2} \pi^{2} R^{2} \lesssim 3
$$

corresponding to $H<10 \mathrm{eV}$ (i.e. $T_{\mathrm{RH}}<10^{5} \mathrm{GeV}$ ) for $R \sim 10 \mathrm{~nm}$. Note that it is not possible here to straightforwardly interpret the decrease of the Planck mass during inflation in terms of the tensor-to-scalar ratio, as we will discuss in the following.

The first relation in (4.19) can be written as

$$
H^{2}=\frac{4 k^{2} \epsilon_{0}}{3\left(f_{s, \pi}^{2}+f_{s, \pi}-1\right)} \approx \frac{3 \epsilon_{0}}{\pi^{2} R^{2}}=\frac{2}{3} k \pi R \times \frac{\rho_{0}}{\left.3 M_{\mathrm{Pl}, \mathrm{eff}}^{2}\right|_{H=0} ^{\mathrm{LD}}},
$$

where $\rho_{0}$ denotes the surplus energy density on the visible brane and we have expanded in $k \pi R \gg 1$ to simplify the result. Note that we do not recover the standard expansion law on the visible brane unlike in the LED or RS case with inflation. Just like in the cases of "remote" inflation in the RS model and the special case of Nihei-Kaloper-Kim-Kim, the expansion law of the visible brane is non-standard. The origin of the non-standard expansion law here is the necessary relation between the surplus energy densities on the IR and UV branes, manifest in the second equation of (4.19). Such a relation is, of course, inconsistent with the picture of having the inflaton sector confined to one of the branes. Restoring the individual contributions of the two brane energy densities to the expansion one can write

$$
H^{2} \approx \frac{\rho_{0}}{\left.3 M_{\mathrm{Pl}, \mathrm{eff}}^{2}\right|_{H=0} ^{\mathrm{LD}}}+\frac{8}{27} k^{3} \pi^{3} R^{3} \times \frac{\rho_{\pi}}{\left.3 M_{\mathrm{Pl}, \mathrm{eff}}^{2}\right|_{H=0} ^{\mathrm{LD}}} .
$$

This shows that if one would ignore the required interrelation of energy densities and simply set $\rho_{\pi} \rightarrow 0$ the standard expansion law on the visible brane would be recovered. However, such an ad-hoc prescription is inconsistent with our solution, in particular with 
the relation (4.19). In this sense the common wisdom, that a stabilized radius leads to a standard inflation law on the brane, does not hold for the Linear Dilaton model.

Clearly, the requirements to apply the analysis of [1] are not fulfilled here. In particular, it is not possible to assume that the energy density that drives inflation - i.e. the inflaton and its potential - is confined to the visible brane. A dedicated study would be required to track the impact of the non-standard expansion law on the observable scalar and tensor mode perturbations after inflation.

Let us remark that the physical origin of the relation between the brane energy densities in (4.19), which leads to the non-standard expansion law, is the dilaton degree of freedom. In the present case, the dilaton dynamics does not decouple from the system even in low energy limit so that the correlation of the two brane energy densities still holds. In a sense, the situation is similar to the NKKK case in the RS model. In that case, the radion is massless so that the (55) Einstein equation does not decouple from the system. This in turn requires the brane energy density relation (3.38) for a given radius $R$, which leads to the non-standard expansion law (3.39). Just as the correlation (3.38) becomes irrelevant when the radion is heavy in the RS model, also the relation (4.19) in the Linear Dilaton model will be broken if the dilaton gets massive. We will see this to be the case in the next subsection where we introduce additional brane localized dilaton potentials, which make the dilaton fluctuations over the background solution heavy enough to decouple them from the system in the low energy limit.

So far we have been agnostic about the details of the stabilization mechanism. The most economic way to stabilize the extra dimension in the Linear Dilaton model is to invoke the dilaton field itself [10]. If $S$ experiences strong boundary potentials, the field values $S_{s}(0)=S_{0}$ and $S_{s}(\pi R)=S_{\pi}$ on the branes are fixed, corresponding to two additional boundary conditions. In the static case, the size of the extra dimension then is determined by the relation $[10]$

$$
k \pi R=\frac{3}{2}\left[\exp \left(\frac{S_{\pi}-S_{0}}{3}\right)-1\right],
$$

completely analogous to the usual Goldberger-Wise mechanism [24]. An analogous scheme has been adopted for the expanding case in [23]. However, we find that one has to be very careful in applying the stabilization scheme of the static case for the expanding case. The reason is that the boundary conditions (4.17) are modified by the brane potentials of $S$. This does not affect the solution of the static case, as the modified boundary conditions are automatically fulfilled. In the dynamical case, however, the change is important as we will see in the following.

\subsection{The expanding case with dilaton stabilization}

Let us consider the case that the dilaton itself is used as a stabilizer. The dilaton-stabilized solution is somewhat more elaborate than the stabilization by an additional GoldbergerWise scalar, simply due to the fact that the back reaction of the stabilizing field is fully accounted for in the computation of the metric. Assuming an otherwise empty bulk, the bulk Lagrangian is fully specified and the (55) Einstein equation should be taken into account. In addition, the boundary conditions (4.17) are modified by the brane potentials of $S$. 
The (55) equation in the bulk is given by

$$
4 f^{\prime 2}-4 H^{2}-\frac{1}{9} f^{2} S^{2}+\frac{2}{3} \kappa^{2} \Lambda f^{2} \mathrm{e}^{-2 S / 3}=0 .
$$

Stabilization can be achieved by imposing strong boundary potentials for $S$ which can be modeled in (4.1) by the choice

$$
\begin{aligned}
& \mathcal{L}_{0}=-\rho_{0}-\xi_{0} M^{4}\left(S_{0}-S(z)\right)^{2}, \\
& \mathcal{L}_{\pi}=-\rho_{\pi}-\xi_{\pi} M^{4}\left(S_{\pi}-S(z)\right)^{2} .
\end{aligned}
$$

Here, $\xi_{0, \pi}$ are dimensionless parameters that characterize the strength of the respective potential.

This modifies the boundary conditions (4.6) and (4.7) at the respective position $z_{0}=$ $\{0, \pi R\}$ to

$$
\begin{aligned}
& \frac{f^{\prime}\left(z_{0}^{+}\right)-f^{\prime}\left(z_{0}^{-}\right)}{f\left(z_{0}\right)}=-\frac{\kappa^{2}}{3} \mathrm{e}^{-S\left(z_{0}\right) / 3}\left[\rho_{z_{0}}+\xi_{z_{0}} M^{4}\left(S_{z_{0}}-S\left(z_{0}\right)\right)^{2}\right], \\
& S^{\prime}\left(z_{0}^{+}\right)-S^{\prime}\left(z_{0}^{-}\right)=-\kappa^{2} \mathrm{e}^{-S\left(z_{0}\right) / 3}\left[\rho_{z_{0}}+\xi_{z_{0}} M^{4}\left(S_{z_{0}}-S\left(z_{0}\right)\right)^{2}+6 \xi_{z_{0}} M^{4}\left(S_{z_{0}}-S\left(z_{0}\right)\right)\right] .
\end{aligned}
$$

Here, we take the boundary potential parameters $S_{0}$ and $S_{\pi}$ to be the same for the static and the dynamic case.

In the static case, the solutions (4.11) are compatible with the modified boundary conditions if the additional constraints $S_{s}(0)=S_{0}$ and $S_{s}(\pi R)=S_{\pi}$ are fulfilled. Furthermore, the (55) Einstein equation is automatically fulfilled. Thus, the original static case solution is completely consistent also with the assumption of dilaton stabilization and one eventually arrives at (4.26) which determines the stabilized size of the extra dimension.

In the dynamical case, by contrast, the assumption of dilaton stabilization affects the final form of the solution of $f$ and $S$. The bulk solution is still given perturbatively by the ansatz (4.13) with the general solution (4.16). As the bulk Lagrangian is fully specified we require that the (55) equation is solved to linear order in $\delta=H^{2} / k^{2}$, which is the case only if $c_{2}=0$. As above, the boundary conditions are required to be fulfilled to leading order in $\delta$. While the boundary conditions for $f$ are unchanged at leading order in $\delta$, it is evident that the boundary conditions for $S$ in (4.30b) are modified as compared to (4.17). Deviations of the brane tensions compared to the static case should again be small, i.e. $\eta_{0}=-1+\epsilon_{0}$ and $\eta_{\pi}=1+\epsilon_{\pi}$ with $\epsilon_{0, \pi} \ll 1$. Finally, we assume that the boundary potentials are strong, $\xi_{0, \pi} M \gg k,{ }^{6}$ and require again the physical condition $S(0)=0$. Altogether, a consistent solution is given by

$$
\begin{aligned}
& c_{1}=\frac{243}{2} f_{s, \pi}^{3} \frac{\epsilon_{0}+\epsilon_{\pi}}{\epsilon_{0}+\epsilon_{\pi} f_{s, \pi}^{3}}, \quad c_{2}=0, \\
& c_{3}=\frac{27}{2}\left(1-f_{s, \pi}^{3}\right) \frac{\epsilon_{0}}{\epsilon_{0}+\epsilon_{\pi} f_{s, \pi}^{3}}, \quad \text { and } \quad \delta=\frac{4}{3} \frac{\epsilon_{0}+\epsilon_{\pi} f_{s, \pi}^{3}}{f_{s, \pi}^{3}-1} .
\end{aligned}
$$

\footnotetext{
${ }^{6}$ Since both $\xi$ and $k$ are dilaton shift symmetry breaking parameters, one can control the relative size of $k$ compared to $\xi M$.
} 


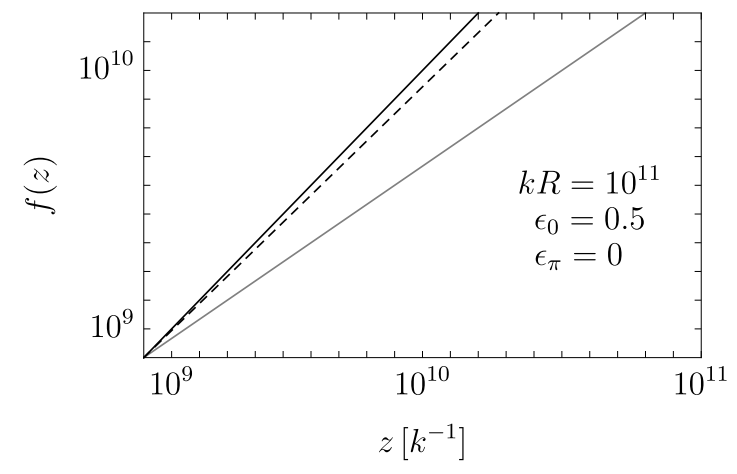

(a)

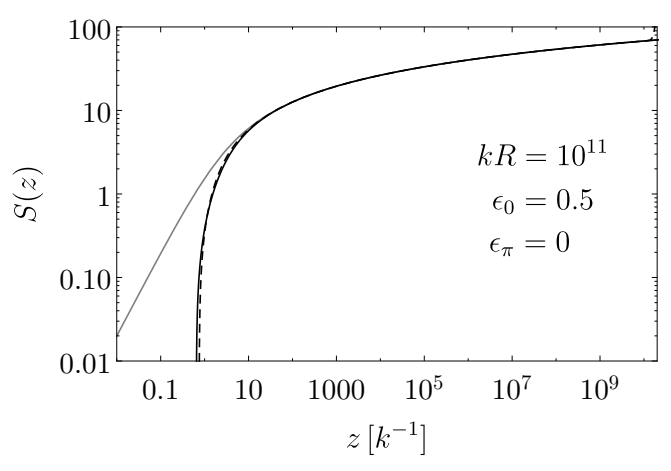

(b)

Figure 5. Metric warping (a) and Dilaton profile (b) of the Linear Dilaton model with inflation in the case where the dilaton itself serves as a stabilizer. Next to our perturbative results (black) we also show a numerical solution (black, dashed) and the static case solution (gray). For the chosen parameters, the resulting Hubble rate is $H \approx 8.5 \times 10^{-18} k \approx 8.5 \times 10^{-15} \mathrm{GeV}$ corresponding to a reheating temperature around the electroweak scale (assuming maximally efficient reheating).

The solution for the metric warping and dilaton profile is fully specified by this. Note that there is no constraint on the relative tensions of the two branes in this case, meaning that $\epsilon_{0}$ and $\epsilon_{\pi}$ can be varied independently. We note that the last relation of (4.31) can be written as

$$
H^{2}=\frac{\rho_{0}}{\left.3 M_{\mathrm{Pl}, \mathrm{eff}}^{2}\right|_{H=0} ^{\mathrm{LD}}}+\frac{f_{s, \pi}^{3} \rho_{\pi}}{\left.3 M_{\mathrm{Pl}, \mathrm{eff}}^{2}\right|_{H=0} ^{\mathrm{LD}}} .
$$

For the case $\rho_{\pi}=0$ corresponding to $\epsilon_{\pi}=0$ the standard expansion law on the visible brane is recovered. In the following we limit ourselves to this case, i.e. we assume that inflation is driven from a surplus energy density located solely on the visible brane. The solution for the metric warping then is

$$
f(z)=1+\frac{2 k}{3}|z|+H^{2} \pi^{3} R^{3} k^{2}|z| \frac{4\left(-9+6 k|z|+4 k^{2} z^{2}\right)}{27(3+2 k|z|)^{2}}+\text { h.o. },
$$

while the dilaton profile at leading order is given by

$$
S(z)=3 \ln \left(1+\frac{2 k}{3}|z|\right)-H^{2} \pi^{3} R^{3} k^{2}|z| \frac{16(3+k|z|)}{(3+2 k|z|)^{3}}+\text { h.o. . }
$$

As before h.o. denotes terms of higher order in $H^{2} / k^{2} \ll 1$ or lower order in $k \pi R \gg 1$. The solutions are displayed in figure 5 together with a numerical solution.

Given the metric warping $f(z)$, the effective Planck mass during inflation can be computed to be

$$
M_{\mathrm{Pl}, \mathrm{eff}}^{2}=\left.M_{\mathrm{Pl}, \mathrm{eff}}^{2}\right|_{H=0} ^{\mathrm{LD}} \times\left(1+\frac{4}{9} H^{2} k \pi^{3} R^{3}+\text { h.o. }\right) .
$$

We note that the Planck mass is enhanced during inflation meaning that the strength of gravity is reduced. Since inflation here is solely driven from the visible brane, the simplifying assumptions of [1] hold and we can interpret our result in terms of the inflationary 
tensor-to-scalar ratio. Since gravity is weakened during inflation the amplitude of tensor modes is reduced.

There is no upper bound on $H$ from perturbativity requirement on gravity. It is noteworthy, however, that the corrections to the static case are proportional to $(H / k)^{2}(k R)^{3}$ in contrast to all other models above, where the $k$ dependence cancels and the corrections were proportional to powers of $(H R)$ only. This does not modify the conclusion of [1], that the $4 \mathrm{D}$ consistency relation $\mathcal{P}_{\mathrm{T}}(\ell) / \mathcal{P}_{\mathrm{S}}(\ell)=-4 n_{\mathrm{T}}$ also holds in the $5 \mathrm{D}$ case.

The extra dimension is stabilized by the dilaton at a size $R$, which is determined by the transcendental relation

$$
S_{\pi}-S_{0}=3 \ln \left(1+\frac{2}{3} k \pi R\right)-\frac{6 \epsilon_{0}}{3+2 k \pi R}-2 \epsilon_{\pi} .
$$

Clearly, this corresponds to (4.26) of the static case. For our particular case of interest $\left(\epsilon_{\pi}=0\right)$ the corrections to the radius with respect to the static case result (4.26) are $\mathcal{O}(k R)^{-1}$ and, thus, completely negligible.

\subsection{Comparison to results in the literature}

Inflation in the Linear Dilaton model has recently also been studied by Kehagias and Riotto (KR) [23]. They have investigated inflation in the LD model under the assumption that the Standard Model and the inflaton both reside on the UV brane and have found that the tensor modes are suppressed. Our setting is conceptually different because we consider the case that the visible brane, which hosts the Standard Model and inflaton fields, is the IR brane such that the gauge-hierarchy problem can also be solved. We also discuss the difference between stabilizing the extra dimension by the dilaton compared to the conventional Goldberger-Wise stabilization. Investigating the setup considered by KR we find qualitative agreement (i.e. suppression of the tensor modes) but we were not able to quantitatively reproduce their results on the metric warping, dilaton profile, and the effective Planck mass.

\section{Conclusions}

We have considered the scheme of inflation in theories with extra space dimensions. In this framework some novel questions arise: why do some dimensions inflate while others are frozen? In the framework of UV-complete theories (as e.g. string theory) this question is related to the mechanism of moduli stabilization. Another question concerns the location of the inflaton field (is it a brane- or a bulk-field) and whether the predictions of the inflationary scenario are influenced by the presence of extra dimensions. This is the question discussed in the present paper. We have concentrated our analysis on those situations where the extra dimensions explain the hierarchy between the weak-scale and the Planck scale. In this case the relative strength of gravity varies in the bulk between visible and hidden brane and this can have consequences for the size of inflationary tensor modes, discussed here in detail. Examples under consideration are large extra dimensions (LED), the Randall-Sundrum scenario (RS) and the linear dilaton model (LD). Up to now the discussion concentrated mainly on simplified cases that satisfy the IRB assumption where 
the inflaton sits on the visible brane and where the mechanism of stabilizing the extra dimensions is assumed not to influence the predictions of inflation.

In a first step we have reexamined the IRB case for LED and RS where exact solutions could be obtained. We observed enhanced tensor modes compared to inflationary prediction in four space-time dimensions. We also stress that in these cases we obtain an upper limit on the Hubble scale $H$. The LD case is more complicated due to the presence of an additional bulk field (the dilaton). We are not able to find exact solutions here but can derive a perturbative expansion in $H^{2}$. Within the $\mathrm{LD}$ framework we find that the naive IRB assumption leads to inconsistencies. Contributions from the hidden brane (or bulk) are needed to obtain the conventional inflationary scenario. This leads us to a scheme of "remote" inflation, where inflation is (partially) driven by energies on the hidden brane.

Motivated by this observation we reconsidered also the RS case beyond the IRB assumption and the properties of (partially) remote inflation. We provide a general class of inflationary solutions for remote inflation that include some specific cases (as NKKK) discussed earlier. Depending on the specific situations, tensor modes could be enhanced or reduced. The consequences for the tensor-to-scalar ratio are not known yet as this would require more calculations beyond the ones given in this paper. In some cases we find an upper limit on the scale of $H$ similar to that found in the IRB case. The analysis of remote inflation in the LD case leads to similar results. The calculation is performed perturbatively in $H^{2}$ and supported by a full numerical solution. Still it would be desirable to extend this to an exact solution as we had derived in the LED and RS case.

The complications in the LD case come from the presence of the additional dilaton field, and this opens new possibilities. One could use the dilaton to stabilize the size of the extra dimension within the scheme itself (without the need for additional stabilizer fields). We have discussed this situation in detail and found the surprising result that this scheme can be made consistent with the IRB assumption (with the inflaton field on the IR brane). In this scheme one obtains a reduced tensor-to-scalar ratio while there is no upper limit on $H$.

The presence of extra dimensions can have strong effects on the prediction of inflationary models, especially in those cases where extra dimensions provide a solution to the weak-scale hierarchy problem. This is an exciting situation in view of new observations concerning the tensor-to-scalar ratio of fluctuations of the cosmic microwave background.

\section{Acknowledgments}

We thank Stefan Förste for useful discussions. AT also thanks Zhongyi Zhang for useful discussions. This work has been supported by the German Science Foundation (DFG) within the SFB-Transregio TR33 "The Dark Universe".

\section{A Agreement with earlier results on the RS1 case}

We show that our results in (3.25)-(3.27) on the Hubble rate, metric warping, and effective Planck mass for the RS1 case are in agreement with the results obtained by Giudice et al. [1]. 
In order to make the connection with the results of [1] one has to recall that they work in the " $\pi$-frame" while we have chosen to put the visible brane at $z=0$ throughout this note. In order to reproduce the metric warping in eq. (94) of [1] one performs the coordinate transformation $z \rightarrow-z+\pi R$ in (3.26) to obtain

$$
\left.f^{2}(z)\right|_{\pi-\text { frame }}=\frac{H^{2}}{\mu^{2}} \mathrm{e}^{\mu z}+\left[1+\frac{H^{2}}{\mu^{2}}\left(\frac{2 \omega-1}{\omega}\right)\right] \frac{\mathrm{e}^{-\mu z}}{\omega}-\frac{2 H^{2}}{\mu^{2}} .
$$

Using the identities $K \equiv \mu / 2$ and $\Omega^{2} \equiv \omega$ one then finds

$$
\left.n^{2}(z) \equiv \Omega^{2} f^{2}(z)\right|_{\pi-\text { frame }}=\frac{\Omega^{2} H^{2}}{4 K^{2}} \mathrm{e}^{2 K z}+\left[1+\frac{H^{2}\left(2 \Omega^{2}-1\right)}{4 \Omega^{2} K^{2}}\right] \mathrm{e}^{-2 K z}-\frac{\Omega^{2} H^{2}}{2 K^{2}},
$$

in perfect agreement with [1]. Using the same identities the Hubble rate (3.25) can be written as

$$
H^{2}=\frac{\epsilon_{0} \mu^{2}}{2} \frac{\omega}{1-\omega}=\frac{\kappa^{2} \mu \rho_{0}}{6} \frac{\omega}{1-\omega} \stackrel{[1]}{=} \frac{K \rho_{0}}{3 M^{3}} \frac{\Omega^{2}}{1-\Omega^{2}} .
$$

This agrees with eq. (95) of [1] up to a factor of $\Omega^{2}$ which is simply due to their choice of $M_{*} \sim M_{\mathrm{Pl}}$ as the fundamental scale.

\section{B Dictionary of different coordinate conventions}

In the discussion of the Linear Dilaton model multiple coordinate conventions have been used in the literature. The coordinates in this work are chosen such that the extra dimension is flat implying that $z$ denotes the proper length of the extra dimension. A different natural choice of coordinates other than ours is given by

$$
y(z)=\frac{3}{2 k} \ln \left(1+\frac{2}{3} k z\right), \quad \Longleftrightarrow \quad z(y)=\frac{3}{2 k}\left(\mathrm{e}^{2 k y / 3}-1\right) .
$$

In this basis the metric of the static case is given by

$$
\mathrm{d} s^{2}=\mathrm{e}^{4 k y / 3}\left(\eta_{\mu \nu} \mathrm{d} x^{\mu} \mathrm{d} x^{\nu}-\mathrm{d} y^{2}\right)
$$

while the dilaton profile is given by

$$
S(y) \equiv \phi(y)=2 k y
$$

The translation of all of our results to this basis is straightforward. In particular, we emphasize that our solutions (4.16) as well as the specifically determined coefficients (4.18) and (4.31) are stated in a form which is independent of the chosen basis. Depending on the desired basis the explicit form of the solutions can be obtained by using $f_{s}(y)=\mathrm{e}^{2 k y / 3}$ instead of $f_{s}(z)=1+2 k z / 3$.

Open Access. This article is distributed under the terms of the Creative Commons Attribution License (CC-BY 4.0), which permits any use, distribution and reproduction in any medium, provided the original author(s) and source are credited. 


\section{References}

[1] G.F. Giudice, E.W. Kolb, J. Lesgourgues and A. Riotto, Transdimensional physics and inflation, Phys. Rev. D 66 (2002) 083512 [hep-ph/0207145] [INSPIRE].

[2] D. Langlois, R. Maartens and D. Wands, Gravitational waves from inflation on the brane, Phys. Lett. B 489 (2000) 259 [hep-th/0006007] [INSPIRE].

[3] A.V. Frolov and L. Kofman, Gravitational waves from brane world inflation, hep-th/0209133 [INSPIRE].

[4] R. Maartens and K. Koyama, Brane-world gravity, Living Rev. Rel. 13 (2010) 5 [arXiv:1004.3962] [INSPIRE].

[5] N. Arkani-Hamed, S. Dimopoulos and G.R. Dvali, The hierarchy problem and new dimensions at a millimeter, Phys. Lett. B 429 (1998) 263 [hep-ph/9803315] [INSPIRE].

[6] N. Arkani-Hamed, S. Dimopoulos and G.R. Dvali, Phenomenology, astrophysics and cosmology of theories with submillimeter dimensions and TeV scale quantum gravity, Phys. Rev. D 59 (1999) 086004 [hep-ph/9807344] [INSPIRE].

[7] L. Randall and R. Sundrum, A large mass hierarchy from a small extra dimension, Phys. Rev. Lett. 83 (1999) 3370 [hep-ph/9905221] [INSPIRE].

[8] I. Antoniadis, S. Dimopoulos and A. Giveon, Little string theory at a TeV, JHEP 05 (2001) 055 [hep-th/0103033] [INSPIRE].

[9] I. Antoniadis, A. Arvanitaki, S. Dimopoulos and A. Giveon, Phenomenology of TeV little string theory from holography, Phys. Rev. Lett. 108 (2012) 081602 [arXiv:1102.4043] [INSPIRE].

[10] P. Cox and T. Gherghetta, Radion dynamics and phenomenology in the linear dilaton model, JHEP 05 (2012) 149 [arXiv:1203.5870] [INSPIRE].

[11] M. Baryakhtar, Graviton phenomenology of linear dilaton geometries, Phys. Rev. D 85 (2012) 125019 [arXiv:1202.6674] [INSPIRE].

[12] J.E. Kim, H.P. Nilles and M. Peloso, Completing natural inflation, JCAP 01 (2005) 005 [hep-ph/0409138] [INSPIRE].

[13] K. Choi, H. Kim and S. Yun, Natural inflation with multiple sub-Planckian axions, Phys. Rev. D 90 (2014) 023545 [arXiv:1404.6209] [INSPIRE].

[14] K. Choi and S.H. Im, Realizing the relaxion from multiple axions and its UV completion with high scale supersymmetry, JHEP 01 (2016) 149 [arXiv: 1511.00132] [INSPIRE].

[15] D.E. Kaplan and R. Rattazzi, Large field excursions and approximate discrete symmetries from a clockwork axion, Phys. Rev. D 93 (2016) 085007 [arXiv: 1511.01827] [INSPIRE].

[16] G.F. Giudice and M. McCullough, A clockwork theory, JHEP 02 (2017) 036 [arXiv: 1610.07962] [INSPIRE].

[17] N. Craig, I. Garcia Garcia and D. Sutherland, Disassembling the clockwork mechanism, JHEP 10 (2017) 018 [arXiv:1704.07831] [INSPIRE].

[18] G.F. Giudice and M. McCullough, Comment on "disassembling the clockwork mechanism", arXiv: 1705.10162 [INSPIRE].

[19] T. Nihei, Inflation in the five-dimensional universe with an orbifold extra dimension, Phys. Lett. B 465 (1999) 81 [hep-ph/9905487] [INSPIRE]. 
[20] N. Kaloper, Bent domain walls as brane worlds, Phys. Rev. D 60 (1999) 123506 [hep-th/9905210] [INSPIRE].

[21] H.B. Kim and H.D. Kim, Inflation and gauge hierarchy in Randall-Sundrum compactification, Phys. Rev. D 61 (2000) 064003 [hep-th/9909053] [INSPIRE].

[22] L. Randall and R. Sundrum, An alternative to compactification, Phys. Rev. Lett. 83 (1999) 4690 [hep-th/9906064] [INSPIRE].

[23] A. Kehagias and A. Riotto, Clockwork inflation, Phys. Lett. B 767 (2017) 73 [arXiv: 1611.03316] [INSPIRE].

[24] W.D. Goldberger and M.B. Wise, Modulus stabilization with bulk fields, Phys. Rev. Lett. 83 (1999) 4922 [hep-ph/9907447] [INSPIRE].

[25] C. Csáki, M. Graesser, L. Randall and J. Terning, Cosmology of brane models with radion stabilization, Phys. Rev. D 62 (2000) 045015 [hep-ph/9911406] [INSPIRE].

[26] P. Kanti, I.I. Kogan, K.A. Olive and M. Pospelov, Cosmological three-brane solutions, Phys. Lett. B 468 (1999) 31 [hep-ph/9909481] [INSPIRE].

[27] P. Kanti, K.A. Olive and M. Pospelov, Static solutions for brane models with a bulk scalar field, Phys. Lett. B 481 (2000) 386 [hep-ph/0002229] [INSPIRE].

[28] J. Lesgourgues, S. Pastor, M. Peloso and L. Sorbo, Cosmology of the Randall-Sundrum model after dilaton stabilization, Phys. Lett. B 489 (2000) 411 [hep-ph/0004086] [INSPIRE].

[29] P. Binetruy, C. Deffayet, U. Ellwanger and D. Langlois, Brane cosmological evolution in a bulk with cosmological constant, Phys. Lett. B 477 (2000) 285 [hep-th/9910219] [INSPIRE].

[30] W. Israel, Singular hypersurfaces and thin shells in general relativity, Nuovo Cim. B 44S10 (1966) 1 [Erratum ibid. B 48 (1967) 463] [INSPIRE].

[31] P. Binetruy, C. Deffayet and D. Langlois, Nonconventional cosmology from a brane universe, Nucl. Phys. B 565 (2000) 269 [hep-th/9905012] [InSPIRE].

[32] L.H. Ford, Gravitational particle creation and inflation, Phys. Rev. D 35 (1987) 2955 [INSPIRE].

[33] L.P. Grishchuk and Yu. V. Sidorov, Squeezed quantum states of relic gravitons and primordial density fluctuations, Phys. Rev. D 42 (1990) 3413 [INSPIRE].

[34] T. Damour and A. Vilenkin, String theory and inflation, Phys. Rev. D 53 (1996) 2981 [hep-th/9503149] [INSPIRE].

[35] M. Giovannini, Gravitational waves constraints on postinflationary phases stiffer than radiation, Phys. Rev. D 58 (1998) 083504 [hep-ph/9806329] [INSPIRE].

[36] C. Gomez, B. Janssen and P.J. Silva, Brane world with bulk horizons, JHEP 04 (2000) 027 [hep-th/0003002] [INSPIRE].

[37] O. Aharony, M. Berkooz, D. Kutasov and N. Seiberg, Linear dilatons, NS five-branes and holography, JHEP 10 (1998) 004 [hep-th/9808149] [INSPIRE].

[38] O. Aharony, A brief review of 'little string theories', Class. Quant. Grav. 17 (2000) 929 [hep-th/9911147] [INSPIRE].

[39] K.A. Meissner and M. Olechowski, General warped solutions in $5 D$ dilaton gravity, Class. Quant. Grav. 20 (2003) 5391 [hep-th/0305170] [INSPIRE]. 